MATHEMATICS OF COMPUTATION

Volume 74, Number 249, Pages 455-484

S $0025-5718(04) 01644-8$

Article electronically published on May 18, 2004

\title{
VISIBLE EVIDENCE \\ FOR THE BIRCH AND SWINNERTON-DYER CONJECTURE FOR MODULAR ABELIAN VARIETIES OF ANALYTIC RANK ZERO
}

\author{
AMOD AGASHE AND WILLIAM STEIN, \\ WITH AN APPENDIX BY J. CREMONA AND B. MAZUR
}

\begin{abstract}
This paper provides evidence for the Birch and Swinnerton-Dyer conjecture for analytic rank 0 abelian varieties $A_{f}$ that are optimal quotients of $J_{0}(N)$ attached to newforms. We prove theorems about the ratio $L\left(A_{f}, 1\right) / \Omega_{A_{f}}$, develop tools for computing with $A_{f}$, and gather data about certain arithmetic invariants of the nearly 20,000 abelian varieties $A_{f}$ of level $\leq 2333$. Over half of these $A_{f}$ have analytic rank 0 , and for these we compute upper and lower bounds on the conjectural order of $\amalg\left(A_{f}\right)$. We find that there are at least 168 such $A_{f}$ for which the Birch and Swinnerton-Dyer conjecture implies that $\amalg\left(A_{f}\right)$ is divisible by an odd prime, and we prove for 37 of these that the odd part of the conjectural order of $\amalg\left(A_{f}\right)$ really divides \# $\amalg\left(A_{f}\right)$ by constructing nontrivial elements of $\amalg\left(A_{f}\right)$ using visibility theory. We also give other evidence for the conjecture. The appendix, by Cremona and Mazur, fills in some gaps in the theoretical discussion in their paper on visibility of Shafarevich-Tate groups of elliptic curves.
\end{abstract}

\section{INTRODUCTION}

Let $N$ be a positive integer, and let $f$ be a newform of weight 2 on $\Gamma_{0}(N)$. A construction due to Shimura associates to $f$ an abelian variety quotient $A_{f}$ of $J_{0}(N)$. We say that $A_{f}$ has analytic rank zero if its $L$-function $L\left(A_{f}, s\right)$ is nonzero at $s=1$. In this paper we give evidence for the Birch and SwinnertonDyer conjecture for analytic rank 0 abelian varieties $A_{f}$ of arbitrary dimension. For such abelian varieties, the conjecture asserts that $A_{f}(\mathbf{Q})$ is finite and gives a formula for the order of the Shafarevich-Tate group $\amalg\left(A_{f}\right)$.

Kolyvagin and Logachev proved in [KL89 KL92] that if $L\left(A_{f}, 1\right) \neq 0$, then $A_{f}(\mathbf{Q})$ and $\amalg\left(A_{f}\right)$ are both finite. To the best of our knowledge, Birch and Swinnerton-Dyer's formula for \# $\amalg\left(A_{f}\right)$ has not been completely verified for a single abelian variety $A_{f}$ of dimension greater than one. In [KL92, §1.6] Kolyvagin and Logachev remark that if one were able to compute the height of a certain Heegner point, their methods could be used to find an upper bound on \# $\amalg\left(A_{f}\right)$,

Received by the editor May 17, 2002 and, in revised form, June 9, 2003.

2000 Mathematics Subject Classification. Primary 11G40; Secondary 11F11, 11G10, 14K15, $14 \mathrm{H} 25,14 \mathrm{H} 40$.

Key words and phrases. Birch and Swinnerton-Dyer conjecture, modular abelian variety, visibility, Shafarevich-Tate groups.

(C)2004 American Mathematical Society 
but we have not done this. Instead, in this paper we focus on computing nonzero subgroups of $\amalg\left(A_{f}\right)$ when the conjecture predicts that $\amalg\left(A_{f}\right)$ is nonzero.

Inspired by work of Cremona and Mazur (see $[\mathrm{CM}]$ ), we had the idea of reversing their methods and of proving, in some cases, that \# $\amalg\left(A_{f}\right)$ is at least as big as predicted by the Birch and Swinnerton-Dyer conjecture. Instead of assuming that $\amalg\left(A_{f}\right)$ is as predicted by the conjecture and trying to understand whether or not it is visible in $J_{0}(N)$, we prove a theorem (see AS02]) that allows us to sometimes construct the odd part of $\amalg\left(A_{f}\right)$ without assuming any conjectures. After developing algorithms that allow us to compute the conjectural order of $\amalg\left(A_{f}\right)$ in most cases, we analyzed the 19608 abelian varieties $A_{f}$ of level $\leq 2333$, and we constructed the tables of Section 5. This resulted in the first systematic experimental evidence for the Birch and Swinnerton-Dyer conjecture for modular abelian varieties of dimension greater than 2 (see FpS:01 for dimension 2).

This paper is organized as follows. In Section 2 we review the background of modular abelian varieties and we state the Birch and Swinnerton-Dyer conjecture. Section 3 explains the basic facts about quotients $A_{f}$ of $J_{0}(N)$ that one needs to know in order to compute with them. In Section 4 we discuss a generalization of the Manin constant, derive a formula for the ratio $L\left(A_{f}, 1\right) / \Omega_{A_{f}}$, and bound the denominator of this ratio, thus giving some theoretical evidence towards the

Birch and Swinnerton-Dyer conjecture. Section 5 reports on our construction of a table of 168 rank 0 abelian varieties $A_{f}$ of level $\leq 2333$ such that the Birch and Swinnerton-Dyer conjecture predicts that \# $\amalg\left(A_{f}\right)$ is divisible by an odd prime, and it discusses what we computed to show that for 37 of the $A_{f}$ there are at least as many elements of the odd part of \# $\amalg\left(A_{f}\right)$ as predicted. The part of \# $\amalg\left(A_{f}\right)$ that is coprime to the modular degree of $A_{f}$ (which we define below) is a perfect square, and in the several cases where we could compute the odd part of the conjectured value of \# $\amalg\left(A_{f}\right)$, we found the odd part to be a perfect square, which gives computational evidence for the conjecture. The appendix, written by Cremona and Mazur, fills in some gaps in the theoretical discussion in [CM].

\section{ACKNOWLEDGMENTS}

It is a pleasure to thank Bryan Birch, Robert Coleman, Benedict Gross, Hendrik Lenstra, Dino Lorenzini, Loïc Merel, Bjorn Poonen, Ken Ribet, and John Tate for many helpful comments and discussions. Special thanks go to Barry Mazur for guiding our ideas on visibility and purchasing a powerful computer for the second author, and to Allan Steel and David Kohel at Magma for their crucial computational support.

\section{BACKGROUND AND DEFINITIONS}

2.1. Modular forms. Fix a positive integer $N$. The group

$$
\Gamma_{0}(N)=\left\{\left(\begin{array}{ll}
a & b \\
c & d
\end{array}\right) \in \mathrm{SL}_{2}(\mathbf{Z}): N \mid c\right\}
$$

acts by linear fractional transformations on the extended complex upper halfplane $\mathfrak{h}^{*}$. As a Riemann surface, $X_{0}(N)(\mathbf{C})$ is the quotient $\Gamma_{0}(N) \backslash \mathfrak{h}^{*}$. There is a standard model for $X_{0}(N)$ over $\mathbf{Q}$ (see [Shi94, Ch. 6]), and the Jacobian $J_{0}(N)$ of $X_{0}(N)$ is an abelian variety over $\mathbf{Q}$ of dimension equal to the genus $g$ of $X_{0}(N)$, which is equipped with an action of the Hecke algebra $\mathbf{T}=\mathbf{Z}\left[\ldots T_{n} \ldots\right]$. The space 
$S_{2}\left(\Gamma_{0}(N)\right)$ of cuspforms of weight 2 on $\Gamma_{0}(N)$ is a module over $\mathbf{T}$ and $S_{2}\left(\Gamma_{0}(N)\right) \cong$ $H^{0}\left(X_{0}(N), \Omega_{X_{0}(N)}\right)$ as $\mathbf{T}$-modules.

\subsection{Abelian varieties attached to newforms. A newform}

$$
f=\sum_{n \geq 1} a_{n} q^{n} \in S_{2}\left(\Gamma_{0}(N)\right)
$$

is an eigenvector for $\mathbf{T}$ that is normalized so that $a_{1}=1$ and which lies in the orthogonal complement of the old subspace of $S_{2}\left(\Gamma_{0}(N)\right)$. Let $I_{f}$ denote the annihilator $\operatorname{Ann}_{\mathbf{T}}(f)$ of $f$ in $\mathbf{T}$. Following Shimura [Shi73], attach to $I_{f}$ the quotient

$$
A_{f}=J_{0}(N) / I_{f} J_{0}(N),
$$

which is an abelian variety over $\mathbf{Q}$ of dimension $\left[\mathbf{Q}\left(\ldots, a_{n}, \ldots\right): \mathbf{Q}\right]$, which is equipped with a faithful action of $\mathbf{T} / I_{f}$. Moreover, $A_{f}$ is an optimal quotient of $J_{0}(N)$, in the sense that $A_{f}^{\vee} \rightarrow J_{0}(N)$ is a closed immersion, or equivalently that the kernel of $J_{0}(N) \rightarrow A_{f}$ is connected (see [CS01, Prop. 3.3]).

Also, the complex torus $A_{f}(\mathbf{C})$ fits into the exact sequence

$$
H_{1}\left(X_{0}(N), \mathbf{Z}\right) \rightarrow \operatorname{Hom}\left(S_{2}\left(\Gamma_{0}(N)\right)\left[I_{f}\right], \mathbf{C}\right) \rightarrow A_{f}(\mathbf{C}) \rightarrow 0 .
$$

2.3. The Birch and Swinnerton-Dyer conjecture. The conjecture of Birch and Swinnerton-Dyer makes sense for abelian varieties over fairly general global fields, but we only state a special case. This conjecture involves the $L$-function attached to $A=A_{f}$ :

$$
L(A, s)=\prod_{i=1}^{d} L\left(f^{(i)}, s\right)=\prod_{i=1}^{d}\left(\sum_{n \geq 1} \frac{a_{n}^{(i)}}{n^{s}}\right),
$$

where $f^{(i)}$ is the $i$ th Galois conjugate of $f$ and $a_{n}^{(i)}$ is the $i$ th Galois conjugate of $a_{n}$. It follows from work of Hecke that $L(A, s)$ has an analytic continuation to the whole complex plane and satisfies a functional equation. Birch and Swinnerton-Dyer made the following conjecture, which relates the rank of $A$ to the order of vanishing of $L(A, s)$ at $s=1$.

Conjecture 2.1 (Birch and Swinnerton-Dyer). The Mordell-Weil rank of $A$ is equal to the order of vanishing of $L(A, s)$ at $s=1$, i.e.,

$$
\operatorname{dim}(A(\mathbf{Q}) \otimes \mathbf{Q})=\operatorname{ord}_{s=1} L(A, s) .
$$

Birch and Swinnerton-Dyer also furnished a conjectural formula for the order of the Shafarevich-Tate group

$$
\amalg(A):=\operatorname{ker}\left(H^{1}(\mathbf{Q}, A) \longrightarrow \prod_{\text {all places } v} H^{1}\left(\mathbf{Q}_{v}, A\right)\right) .
$$

(They only made their conjecture for elliptic curves, but Tate Tat66] reformulated it in a functorial way which makes sense for abelian varieties. See also Lan91. $\S$ III.5] for another formulation.) We now state their conjecture in the special case when $L(A, 1) \neq 0$, where KL89, KL92 imply that $\amalg(A)$ is finite. The conjecture involves the Tamagawa numbers $c_{p}$ of $A$ (see Section 3.7) and the canonical volume $\Omega_{A}$ of $A(\mathbf{R})$ (see Section 4.2). 
Conjecture 2.2 (Birch and Swinnerton-Dyer). Suppose $L(A, 1) \neq 0$. Then

$$
\frac{L(A, 1)}{\Omega_{A}}=\frac{\# \amalg(A) \cdot \prod_{p \mid N} c_{p}}{\# A(\mathbf{Q})_{\mathrm{tor}} \cdot \# A^{\vee}(\mathbf{Q})_{\mathrm{tor}}},
$$

where $A^{\vee}$ is the abelian variety dual of $A$.

Remark 2.3. Since $L(A, 1) \neq 0$, finiteness of $\amalg(A)$ and the existence of the CasselsTate pairing imply that \# $\amalg(A)=\# \amalg\left(A^{\vee}\right)$, so Conjecture 2.2 can also be viewed as a formula for \# $\amalg\left(A^{\vee}\right)$.

The algorithms outlined in this paper take advantage of the fact that $A$ is attached to a newform in order to compute the conjectural order of $\amalg(A)$ away from certain bad primes.

\section{ExPlicit APPROACHES TO MODULAR ABELIAN VARIETIES}

We use the algorithms of this section to enumerate the $A_{f}$, to compute information about the invariants of $A_{f}$ that appear in Conjecture 2.2, and to verify the hypothesis of Theorem 3.13 in order to construct nontrivial subgroups of $\amalg\left(A_{f}\right)$. The second author has implemented the algorithms discussed in this paper and made many of them part of the MAGMA computer algebra system [BCP97].

In Section 3.1, we discuss modular symbols, which are the basic tool we use in many of the computations, and in Section 3.2 we discuss how we systematically enumerate modular abelian varieties. There is an analogue for $A_{f}$ of the usual elliptic-curve modular degree, which we discuss in Section 3.3 and which we use to rule out the existence of visible elements of $\amalg\left(A_{f}\right)$ of a certain order. In Section 3.4 we describe how to compute the intersection of two abelian varieties, which will be needed to verify the hypothesis of Theorem 3.13 In Sections 3.5] and 3.6, we describe standard methods for bounding the torsion subgroup of an abelian variety above and below. Section [3.7 reviews an algorithm for computing the odd part of the Tamagawa number $c_{p}$ when $p \| N$, and it discusses the Lenstra-Oort bound in the case when $p^{2} \mid N$.

Unless otherwise stated, $f$ is a newform, $I_{f}$ its annihilator, and $A=A_{f}$ is the corresponding optimal quotient of $J_{0}(N)$.

3.1. Modular symbols. Modular symbols are crucial to many algorithms for computing with modular abelian varieties, because they can be used to construct a finite presentation for $H_{1}\left(X_{0}(N), \mathbf{Z}\right)$ in terms of paths between elements of $\mathbf{P}^{1}(\mathbf{Q})=\mathbf{Q} \cup\{\infty\}$. They were introduced by Birch [Bir71] and studied by Manin, Mazur, Merel, Cremona, and others.

Let $\mathfrak{M}_{2}$ be the free abelian group with basis the set of all symbols $\{\alpha, \beta\}$, with $\alpha, \beta \in \mathbf{P}^{1}(\mathbf{Q})$, modulo the three-term relations

$$
\{\alpha, \beta\}+\{\beta, \gamma\}+\{\gamma, \alpha\}=0
$$

and modulo any torsion. The group $\mathrm{GL}_{2}(\mathbf{Q})$ acts on the left on $\mathfrak{M}_{2}$ by

$$
g\{\alpha, \beta\}=\{g(\alpha), g(\beta)\},
$$

where $g$ acts on $\alpha$ and $\beta$ by a linear fractional transformation. The space $\mathfrak{M}_{2}\left(\Gamma_{0}(N)\right)$ of modular symbols for $\Gamma_{0}(N)$ is the quotient of $\mathfrak{M}_{2}$ by the subgroup generated by all elements of the form $x-g(x)$, for $x \in \mathfrak{M}_{2}$ and $g$ in $\Gamma_{0}(N)$, modulo any torsion. A modular symbol for $\Gamma_{0}(N)$ is an element of this space, and we frequently denote the equivalence class that defines a modular symbol by giving a representative element. 
Let $\mathfrak{B}_{2}\left(\Gamma_{0}(N)\right)$ be the free abelian group with basis the finite set $\Gamma_{0}(N) \backslash \mathbf{P}^{1}(\mathbf{Q})$. The boundary map $\delta: \mathfrak{M}_{2}\left(\Gamma_{0}(N)\right) \rightarrow \mathfrak{B}_{2}\left(\Gamma_{0}(N)\right)$ sends $\{\alpha, \beta\}$ to $[\beta]-[\alpha]$, where $[\beta]$ denotes the basis element of $\mathfrak{B}_{2}\left(\Gamma_{0}(N)\right)$ corresponding to $\beta \in \mathbf{P}^{1}(\mathbf{Q})$. The cuspidal modular symbols are the kernel $\mathfrak{S}_{2}\left(\Gamma_{0}(N)\right)$ of $\delta$, and the integral homology $H_{1}\left(X_{0}(N), \mathbf{Z}\right)$ is canonically isomorphic to $\mathfrak{S}_{2}\left(\Gamma_{0}(N)\right)$.

Cremona's book [Cre97, §2.2] contains a concrete description of how to compute $\mathfrak{M}_{2}\left(\Gamma_{0}(N)\right) \otimes \mathbf{Q}$ using Manin symbols, which are a finite set of generators for $\mathfrak{M}_{2}\left(\Gamma_{0}(N)\right)$. In general, the easiest way we have found to compute $\mathfrak{M}_{2}\left(\Gamma_{0}(N)\right)$ is to compute $\mathfrak{M}_{2}\left(\Gamma_{0}(N)\right) \otimes \mathbf{Q}$ and then to compute the $\mathbf{Z}$-submodule of $\mathfrak{M}_{2}\left(\Gamma_{0}(N)\right) \otimes \mathbf{Q}$ generated by the Manin symbols.

3.2. Enumerating newforms. Since $X_{0}(N)$ is defined over Q, it is defined over $\mathbf{R}$, so complex conjugation acts on $X_{0}(N)(\mathbf{C})$ and hence on the homology $H_{1}\left(X_{0}(N), \mathbf{Z}\right)$. In terms of modular symbols, complex conjugation acts by sending $\{\alpha, \beta\}$ to $\{-\alpha,-\beta\}$. Let $H_{1}\left(X_{0}(N), \mathbf{Z}\right)^{+}$denote the +1 -eigenspace for the action of the involution induced by complex conjugation, which we can compute using modular symbols. We list all newforms of a given level $N$ by decomposing the new subspace of $H_{1}\left(X_{0}(N), \mathbf{Q}\right)^{+}$under the action of the the Hecke operators and listing the corresponding systems of Hecke eigenvalues (see [Ste02a]). First we compute the characteristic polynomial of $T_{2}$ and use it to break up the new space. We apply this process recursively with $T_{3}, T_{5}, \ldots$ either until we have exceeded the bound coming from [Stu87] (see [AS]) or until we have found a Hecke operator $T_{n}$ whose characteristic polynomial is irreducible.

We order the newforms in a way that extends the ordering in Cre97: First sort by dimension, with smallest dimension first; within each dimension, sort in binary by the signs of the Atkin-Lehner involutions, e.g.,,,,+++++-+-++-- , -++ , etc. When two forms have the same Atkin-Lehner sign sequence, order by $\left|\operatorname{Tr}\left(a_{p}\right)\right|$ with ties broken by taking the positive trace first. We denote a Galoisconjugacy class of newforms by a bold symbol such as $389 \mathrm{E}$, which consists of a level and isogeny class, where $\mathbf{A}$ denotes the first class, $\mathbf{B}$ the second, $\mathbf{E}$ the fifth, BB the 28th, etc. As discussed in [Cre97, p. 5], for certain small levels the above ordering, when restricted to elliptic curves, does not agree with the ordering used in the tables of [Cre97. For example, our 446B is Cremona's 446D.

3.3. The modular degree. Since $A_{f}$ is an optimal quotient, the dual map $A_{f}^{\vee} \rightarrow$ $J_{0}(N)$ is injective and the composite $\theta_{f}: A_{f}^{\vee} \rightarrow A_{f}$ has finite degree. The map $\theta_{f}$ is a polarization, so $\operatorname{deg}\left(\theta_{f}\right)$ is a perfect square (see Lemma 3.14). The modular degree of $A_{f}$ is the square root of the degree of $\theta_{f}$ :

$$
\operatorname{moddeg}\left(A_{f}\right)=\sqrt{\operatorname{deg}\left(\theta_{f}\right)} \text {. }
$$

When $\operatorname{dim} A_{f}=1, \operatorname{moddeg}\left(A_{f}\right)$ is the usual modular degree, i.e., the degree of $X_{0}(N) \rightarrow A_{f}$.

If $M$ is an abelian group, let $M^{*}=\operatorname{Hom}_{\mathbf{Z}}(M, \mathbf{Z})$. The Hecke algebra acts in a natural way on $H_{1}\left(X_{0}(N), \mathbf{Z}\right)$ and $H_{1}\left(X_{0}(N), \mathbf{Z}\right)^{*}$, and we have a natural restriction map

$$
r_{f}: H_{1}\left(X_{0}(N), \mathbf{Z}\right)^{*}\left[I_{f}\right] \rightarrow\left(H_{1}\left(X_{0}(N), \mathbf{Z}\right)\left[I_{f}\right]\right)^{*} .
$$

The following proposition leads to an algorithm for computing the modular degree.

Proposition 3.1. $\operatorname{coker}\left(r_{f}\right) \cong \operatorname{ker}\left(\theta_{f}\right)$, so $\operatorname{moddeg}\left(A_{f}\right)=\sqrt{\# \operatorname{coker}\left(r_{f}\right)}$. 
The proposition is proved in [KS00. The proof makes use of the Abel-Jacobi theorem, which realizes the Jacobian $J_{0}(N)(\mathbf{C})$ as a complex torus:

$$
0 \rightarrow H_{1}\left(X_{0}(N), \mathbf{Z}\right) \rightarrow \operatorname{Hom}\left(S_{2}\left(\Gamma_{0}(N)\right), \mathbf{C}\right) \rightarrow J_{0}(N)(\mathbf{C}) \rightarrow 0,
$$

where $H_{1}\left(X_{0}(N), \mathbf{Z}\right)$ is embedded as a lattice of full rank in the complex vector space $\operatorname{Hom}\left(S_{2}\left(\Gamma_{0}(N)\right), \mathbf{C}\right)$ using the integration pairing, and this description of $J_{0}(N)(\mathbf{C})$ is compatible with the action of Hecke operators.

3.4. Intersecting complex tori. Let $V$ be a finite dimensional complex vector space and let $\Lambda$ be a lattice in $V$, so that $T=V / \Lambda$ is a complex torus. Suppose that $V_{A}$ and $V_{B}$ are subspaces of $V$ such that $\Lambda_{A}=V_{A} \cap \Lambda$ and $\Lambda_{B}=V_{B} \cap \Lambda$ are lattices in $V_{A}$ and $V_{B}$, respectively.

Proposition 3.2. If $A \cap B$ is finite, then there is an isomorphism

$$
A \cap B \cong\left(\frac{\Lambda}{\Lambda_{A}+\Lambda_{B}}\right)_{\text {tor } .}
$$

Proof. Extend the exact sequence

$$
0 \rightarrow A \cap B \rightarrow A \oplus B \stackrel{(x, y) \mapsto x-y}{\longrightarrow} T
$$

to the following diagram:

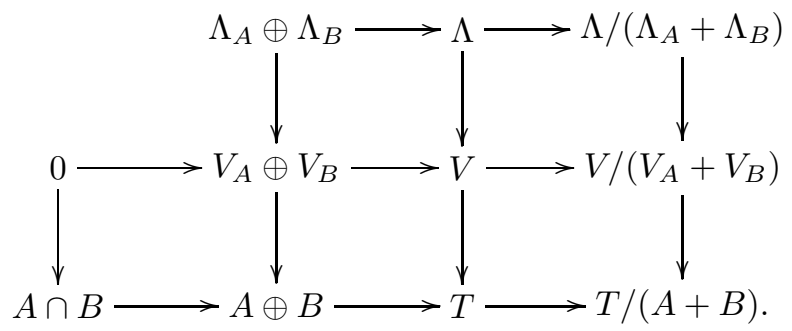

The middle row is exact because $A \cap B$ is finite so $V_{A} \cap V_{B}=0$.

Using the snake lemma, which connects the kernel $A \cap B$ of $A \oplus B \rightarrow T$ to the cokernel of $\Lambda_{A} \oplus \Lambda_{B} \rightarrow \Lambda$, we obtain an exact sequence

$$
0 \rightarrow A \cap B \rightarrow \Lambda /\left(\Lambda_{A}+\Lambda_{B}\right) \rightarrow V /\left(V_{A}+V_{B}\right) .
$$

Since $V /\left(V_{A}+V_{B}\right)$ is a $\mathbf{C}$-vector space, the torsion part of $\Lambda /\left(\Lambda_{A}+\Lambda_{B}\right)$ must map to 0 . No nontorsion in $\Lambda /\left(\Lambda_{A}+\Lambda_{B}\right)$ could map to 0 , because if it did, then $A \cap B$ would not be finite. The proposition follows.

For abelian subvarieties of $J_{0}(N)$ attached to newforms, we use the proposition above as follows. The complex vector space $V=\operatorname{Hom}\left(S_{2}\left(\Gamma_{0}(N)\right), \mathbf{C}\right)$ is the tangent space of $J_{0}(N)(\mathbf{C})$ at the identity. Setting $\Lambda=H_{1}\left(X_{0}(N), \mathbf{Z}\right)$ and considering $\Lambda$ as a lattice in $V$ via the integration pairing, we have $J_{0}(N)(\mathbf{C}) \cong V / \Lambda$. Suppose $f$ and $g$ are nonconjugate newforms, and let $I_{f}$ and $I_{g}$ be their annihilators in the Hecke algebra $\mathbf{T}$, and let $A=A_{f}^{\vee}$ and $B=A_{g}^{\vee}$. Then $V_{A}=V\left[I_{f}\right]$ and $V_{B}=V\left[I_{g}\right]$ are the tangent spaces to $A$ and $B$ at the identity, respectively. The above proposition shows that the group $A \cap B$ is canonically isomorphic to $\left(\Lambda /\left(\Lambda_{A}+\Lambda_{B}\right)\right)_{\text {tor }}$. Here $\Lambda_{A}=\Lambda\left[I_{f}\right]$ and $\Lambda_{B}=\Lambda\left[I_{g}\right]$, because $A_{f}$ and $A_{g}$ are optimal quotients.

The following formula for the intersection of $n$ subtori is obtained in a similar way to that of Proposition 3.2 . 
Proposition 3.3. For $i=1, \ldots, n$, with $n \geq 2$, let $A_{i}=V_{i} / \Lambda_{i}$ be a subtorus of $T=V / \Lambda$, and assume that each pairwise intersection $A_{i} \cap A_{j}$ is finite. Define a linear map

$$
f: V_{1} \times \cdots \times V_{n} \longrightarrow V^{\oplus(n-1)}
$$

by $f\left(x_{1}, \ldots, x_{n}\right)=\left(x_{1}-x_{2}, x_{2}-x_{3}, x_{3}-x_{4}, \ldots, x_{n-1}-x_{n}\right)$. Then

$$
A_{1} \cap \cdots \cap A_{n} \cong\left(\frac{\Lambda^{\oplus(n-1)}}{f\left(\Lambda_{1} \oplus \cdots \oplus \Lambda_{n}\right)}\right)_{\text {tor }} .
$$

3.5. Bounding the torsion from above. In this section we recall the standard upper bound on the order of $\# A(\mathbf{Q})_{\text {tor }}$, and we illustrate its usefulness.

Let $f=\sum a_{n} q^{n}$ be a weight 2 newform on $\Gamma_{1}(N)$ with Nebentypus character $\varepsilon:(\mathbf{Z} / N \mathbf{Z})^{*} \rightarrow \mathbf{C}^{*}$ (recall that $f$ is a form on $\Gamma_{0}(N)$ if and only if $\varepsilon=1$ ), and let $A=A_{f}$ be the corresponding optimal quotient of $J_{1}(N)$, as in Shi73. Shimura proved in [Shi94, Ch. 7] that the local Euler factor of $A_{f}$ at $p$ is

$$
L_{p}\left(A_{f}, s\right)=\prod_{\sigma: K_{f} \hookrightarrow \overline{\mathbf{Q}}} \frac{1}{1-\sigma\left(a_{p}\right) p^{-s}+\sigma(\varepsilon(p)) p^{1-2 s}}
$$

by showing that the characteristic polynomial $F_{p}$ of Frobenius on any $\ell$-adic Tate module of $A_{\mathbf{F}_{p}}($ for $\ell \nmid p N)$ is

$$
F_{p}(X)=\prod_{\sigma: K_{f} \hookrightarrow \overline{\mathbf{Q}}} X^{2}-\sigma\left(a_{p}\right) X+\sigma(\varepsilon(p)) p,
$$

where $K_{f}=\mathbf{Q}\left(\ldots, a_{n}, \ldots\right)$. Let $\mathbf{Q}(\varepsilon)$ be the field generated by the values of $\varepsilon$ (note that $\left.\mathbf{Q}(\varepsilon) \subset K_{f}\right)$, and for any $p \nmid N$ let $G_{p}(X) \in \mathbf{Q}(\varepsilon)[X]$ be the characteristic polynomial of left multiplication by $a_{p}$ on the $\mathbf{Q}(\varepsilon)$-vector space $K_{f}$, which is a polynomial of degree $d^{\prime}=\left[K_{f}: \mathbf{Q}(\varepsilon)\right]$. Then

$$
F_{p}(X)=\operatorname{Norm}_{\mathbf{Q}(\varepsilon) / \mathbf{Q}}\left(X^{d^{\prime}} \cdot G_{p}\left(X+\frac{\varepsilon(p) p}{X}\right)\right),
$$

so

$$
\begin{aligned}
\# A_{\mathbf{F}_{p}}\left(\mathbf{F}_{p}\right) & =\operatorname{deg}\left(1-\operatorname{Frob}_{p}\right)=\left|\operatorname{det}\left(1-\operatorname{Frob}_{p}\right)\right| \\
& =\left|F_{p}(1)\right|=\left|\operatorname{Norm}_{\mathbf{Q}(\varepsilon) / \mathbf{Q}}\left(G_{p}(1+\varepsilon(p) p)\right)\right| .
\end{aligned}
$$

If $p \nmid N$ is odd, standard facts about formal groups imply that the reduction map $A(\mathbf{Q})_{\text {tor }} \rightarrow A_{\mathbf{F}_{p}}\left(\mathbf{F}_{p}\right)$ is injective, so

$$
\# A(\mathbf{Q})_{\text {tor }} \mid \operatorname{gcd}\left\{\# A_{\mathbf{F}_{p}}\left(\mathbf{F}_{p}\right): \text { primes } p \nmid 2 N\right\} \text {. }
$$

Likewise, since $A^{\vee}$ is isogenous to $A$, the same bound applies to $A^{\vee}(\mathbf{Q})_{\text {tor }}$, since $A^{\vee}$ and $A$ have the same $L$-series.

The upper bound is the same for every abelian variety isogenous to $A$, so it is not surprising that it is not sharp in general. For example, let $E$ (resp., $F$ ) be the elliptic curve labeled 30A1 (resp., 30A2) in Cremona's tables [Cre97. Then $E$ and $F$ are isogenous, $E(\mathbf{Q}) \approx \mathbf{Z} / 6 \mathbf{Z}$, and $F(\mathbf{Q}) \approx \mathbf{Z} / 12 \mathbf{Z}$, so

$$
12 \mid \operatorname{gcd}\left\{\# E_{\mathbf{F}_{p}}\left(\mathbf{F}_{p}\right): \text { primes } p \nmid 2 N\right\} .
$$

(Incidentally, since $\# E\left(\mathbf{F}_{5}\right)=12$, the gcd is 12 .) For answers to some related deep questions about this gcd, see Kat81. 
Example 3.4. Let

$$
f=q+(-1+\sqrt{2}) q^{2}+q^{3}+(-2 \sqrt{2}+1) q^{4}-2 \sqrt{2} q^{5}+\cdots \in S_{2}\left(\Gamma_{0}(39)\right)
$$

be the form 39B. Then $G_{5}(X)=X^{2}-8$, so

$$
\# A_{f}(\mathbf{Q})_{\text {tor }} \mid G_{5}(1+5)=28 \text {. }
$$

We find in [FpS:01 that $A_{f}$ is isogenous to the Jacobian $J$ of $y^{2}+\left(x^{3}+1\right) y=$ $-5 x^{4}-2 x^{3}+16 x^{2}-12 x+2$ and that $\# J(\mathbf{Q})=28$. However $A_{f}$ is not isomorphic to $J$ since, as reported in Table 2 of [FpS:01], the Tamagawa numbers of $J$ are $c_{3}=28, c_{13}=1$, whereas the methods of Section 3.7 below show that the Tamagawa numbers of $A_{f}$ are $c_{3}=14, c_{13}=2$. The authors do not know for sure whether $\# A_{f}(\mathbf{Q})=28$, but in Example 3.6 below we show that $14 \mid \# A_{f}(\mathbf{Q})$. (Also, using the computational techniques of this paper, one sees that the Birch and SwinnertonDyer conjecture implies that \# $A_{f}(\mathbf{Q})=28$.)

Example 3.5. Let

$$
f=q+\left(-\zeta_{6}-1\right) q^{2}+\left(2 \zeta_{6}-2\right) q^{3}+\zeta_{6} q^{4}+\left(-2 \zeta_{6}+1\right) q^{5}+\cdots
$$

be one of the two Galois-conjugate newforms in $S_{2}\left(\Gamma_{1}(13)\right)$. This form has character $\varepsilon:(\mathbf{Z} / 13 \mathbf{Z})^{*} \rightarrow \mathbf{C}^{*}$ of order 6 , and $A_{f}=J_{1}(13)$. We have $G_{3}(X)=X-2 \zeta_{6}+2$ and $\varepsilon(3)=-\zeta_{6}$, so

$$
\begin{aligned}
\# J_{1}(13)(\mathbf{Q})_{\text {tor }} \mid \# J_{1}(13)\left(\mathbf{F}_{3}\right) & =\left|\operatorname{Norm}\left(G_{3}\left(1-3 \zeta_{6}\right)\right)\right| \\
& =\left|\operatorname{Norm}\left(-5 \zeta_{6}+3\right)\right|=19 .
\end{aligned}
$$

In fact Ogg proved that $J_{1}(13)(\mathbf{Q})_{\text {tor }} \approx \mathbf{Z} / 19 \mathbf{Z}$ (see $\operatorname{Ogg73}$ and [MT74]).

3.6. Bounding the torsion from below. A cusp $\alpha \in \Gamma_{0}(N) \backslash \mathbf{P}^{1}(\mathbf{Q}) \subset X_{0}(N)$ defines a point $(\alpha)-(\infty) \in J_{0}(N)(\overline{\mathbf{Q}})_{\text {tor }}$. The rational cuspidal subgroup $C$ of $J_{0}(N)(\mathbf{Q})_{\text {tor }}$ generated by $\mathbf{Q}$-rational cusps is of interest because the order of the image of $C$ in $A_{f}(\mathbf{Q})_{\text {tor }}$ provides a lower bound on $\# A_{f}(\mathbf{Q})_{\text {tor }}$. Stevens Ste82. $\S 1.3]$ computed the action of $\mathrm{Gal}(\overline{\mathbf{Q}} / \mathbf{Q})$ on the subgroup of $J_{0}(N)(\overline{\mathbf{Q}})$ generated by all cusps (and for other congruence subgroups besides $\Gamma_{0}(N)$ ). He found that $\operatorname{Gal}(\overline{\mathbf{Q}} / \mathbf{Q})$ acts on the cusps through $\operatorname{Gal}\left(\mathbf{Q}\left(\zeta_{N}\right) / \mathbf{Q}\right) \cong(\mathbf{Z} / N \mathbf{Z})^{*}$ and that $d \in$ $(\mathbf{Z} / N \mathbf{Z})^{*}$ acts by $x / y \mapsto x /\left(d^{\prime} y\right)$, where $d d^{\prime} \equiv 1(\bmod N)$. Thus, e.g., $(0)-(\infty) \in$ $J_{0}(N)(\mathbf{Q})_{\text {tor }}$, and if $N$ is square-free, then all cusps are rational.

To compute the image of $C$ in $A_{f}(\mathbf{Q})_{\text {tor }}$, first make a list of inequivalent cusps using, e.g., the method described in [Cre97, §2.2, p. 17]. Keep only the Q-rational cusps, which can be determined using the result of Stevens above and Cre97. Prop. 2.2.3] (when $N$ is squarefree all cusps are rational). Next compute the subgroup $\mathcal{C}$ of $\mathfrak{M}_{2}\left(\Gamma_{0}(N)\right)$ generated by modular symbols $\{\alpha, \infty\}$, where $\alpha$ is a Q-rational cusp. The image of $C$ in $A_{f}(\mathbf{Q})_{\text {tor }}$ is isomorphic to the image of $\mathcal{C}$ in

$$
P=\Phi_{f}\left(\mathfrak{M}_{2}\left(\Gamma_{0}(N)\right)\right) / \Phi_{f}\left(\mathfrak{S}_{2}\left(\Gamma_{0}(N)\right)\right),
$$

where $\Phi_{f}: \mathfrak{M}_{2}\left(\Gamma_{0}(N)\right) \rightarrow \operatorname{Hom}\left(S_{2}\left(\Gamma_{0}(N)\right)\left[I_{f}\right], \mathbf{C}\right)$ is defined by the integration pairing. To keep everything rational, note that $P$ can be computed using any map with the same kernel as $\Phi_{f}$; for example, such a map can be constructed by finding a basis for $\operatorname{Hom}\left(\mathfrak{M}_{2}\left(\Gamma_{0}(N)\right), \mathbf{Q}\right)\left[I_{f}\right]$ as described at the end of Section 4.2.

Example 3.6. Let the notation be as in Example 3.4 The cusps on $X_{0}(39)$ are represented by $0, \infty,-1 / 9$, and $-4 / 13$, and since $N=39$ is squarefree, these cusps are all rational. Using MAGMA, we find that the image of $C$ in $A_{f}(\mathbf{Q})_{\text {tor }}$ is 
isomorphic to $\mathbf{Z} / 14 \mathbf{Z}$. Thus $A_{f}(\mathbf{Q})_{\text {tor }}$ is isomorphic to one of $\mathbf{Z} / 14 \mathbf{Z}, \mathbf{Z} / 28 \mathbf{Z}$, or $\mathbf{Z} / 14 \mathbf{Z} \times \mathbf{Z} / 2 \mathbf{Z}$, but we do not know which.

Example 3.7. Let

$$
f=q+\frac{1+\sqrt{5}}{2} q^{2}+\frac{1-\sqrt{5}}{2} q^{3}+\frac{5+\sqrt{5}}{2} q^{4}+\cdots \in S_{2}\left(\Gamma_{0}(175)\right)
$$

be the form $175 \mathrm{D}$. The cusps of $X_{0}(175)$ are represented by

$$
0, \infty, \frac{1}{25}, \frac{1}{28}, \frac{1}{30}, \frac{1}{35}, \frac{1}{45}, \frac{1}{60}, \frac{1}{65}, \frac{1}{70}, \frac{1}{105}, \frac{1}{140} .
$$

The Q-rational cusps in this list are $0, \infty, \frac{1}{25}, \frac{1}{28}$, and these generate a subgroup of $A_{f}(\mathbf{Q})_{\text {tor }}$ of order 2. (Incidentally, the group generated by all cusps, both rational and not, is isomorphic to $\mathbf{Z} / 32 \mathbf{Z}$.) Using $a_{p}$ for $p \leq 17$ and the method of the previous section, we see that $\# A_{f}(\mathbf{Q})_{\text {tor }} \mid 4$. The authors do not know if the cardinality is 2 or 4 .

Example 3.8. The form $209 \mathrm{C}$ is

$$
f=q+\alpha q^{2}+\left(1 / 2 \alpha^{4}-\alpha^{3}-5 / 2 \alpha^{2}+4 \alpha+1\right) q^{3}+\left(\alpha^{2}-2\right) q^{4}+\cdots,
$$

where $\alpha^{5}-2 \alpha^{4}-6 \alpha^{3}+10 \alpha^{2}+5 \alpha-4=0$. As above, we find that $\# A_{f}(\mathbf{Q})_{\text {tor }}$ divides 5. The image of the (rational) cuspidal subgroup in $A_{f}(\overline{\mathbf{Q}})_{\text {tor }}$ is isomorphic to $\mathbf{Z} / 5 \mathbf{Z}$, so $A_{f}(\mathbf{Q})_{\text {tor }} \approx \mathbf{Z} / 5 \mathbf{Z}$.

3.7. Tamagawa numbers. Suppose $p \mid N$ and let $\Phi_{A, p}$ denote the component group of $A$ at $p$, which is defined by the following exact sequence:

$$
0 \rightarrow \mathcal{A}_{\mathbf{F}_{p}}^{0} \rightarrow \mathcal{A}_{\mathbf{F}_{p}} \rightarrow \Phi_{A, p} \rightarrow 0,
$$

where $\mathcal{A}_{\mathbf{F}_{p}}$ is the closed fiber of the Néron model of $A$ over $\mathbf{Z}_{p}$ and $\mathcal{A}_{\mathbf{F}_{p}}^{0}$ is the component of $\mathcal{A}_{\mathbf{F}_{p}}$ that contains the identity.

Definition 3.9. The Tamagawa number of $A$ at $p$ is

$$
c_{p}=c_{A, p}=\# \Phi_{A, p}\left(\mathbf{F}_{p}\right) .
$$

When $p \| N$, the second author found a computable formula for $\# \Phi_{A, p}\left(\overline{\mathbf{F}}_{p}\right)$ and (sometimes only up to a power of 2 ) for $\# \Phi_{A, p}\left(\mathbf{F}_{p}\right)$. There is a discussion about how to compute this number in [KS00], and [CS01] contains a proof of the formula. Note also that in this case the Tamagawa number of $A$ at $p$ is the same as the Tamagawa number of $A^{\vee}$ at $p$.

When $p^{2} \mid N$, the authors do not know of an algorithm for computing $c_{p}$. However, in this case Lenstra and Oort (see [LO85]) proved that

$$
\sum_{\ell \neq p}(\ell-1) \operatorname{ord}_{\ell}\left(\# \Phi_{A, p}\left(\overline{\mathbf{F}}_{p}\right)\right) \leq 2 \operatorname{dim}\left(A_{f}\right),
$$

so if $\ell \mid \# \Phi_{A, p}\left(\overline{\mathbf{F}}_{p}\right)$, then $\ell \leq 2 \cdot \operatorname{dim}\left(A_{f}\right)+1$ or $\ell=p$. $\left(\right.$ Here $\operatorname{ord}_{\ell}(x)$ denotes the exponent of the largest power of $\ell$ that divides $x$.)

Example 3.10. Let $f$ be 39B as in Example 3.4, Running the algorithm of [KS00], we find that $c_{3}=14$ and $c_{13}=2$.

Example 3.11. Let $f$ be $\mathbf{1 7 5 D}$ as in Example 3.7. Running the algorithm of KS00, we find that $c_{7}=1$, and the Lenstra-Oort bound implies that the only possible prime divisors of $c_{5}$ are 2,3 , and 5 . 
3.8. Visibility theory. We briefly recall visibility theory, which we will use to construct elements of Shafarevich-Tate groups. Section APPENDIX contains another approach to the results reported in this section, but in the special case of elliptic curves.

Definition 3.12. Let $\iota: A \hookrightarrow J$ be an embedding of abelian varities over $\mathbf{Q}$. The visible subgroup of $\amalg(A)$ with respect to the embedding $\iota$ is

$$
\operatorname{Vis}_{J}(\amalg(A))=\operatorname{Ker}(\amalg(A) \rightarrow \amalg(J)) \text {. }
$$

The following is a special case of Theorem 3.1 of [AS02].

Theorem 3.13. Let $A$ and $B$ be abelian subvarieties of an abelian variety $J$ over $\mathbf{Q}$ such that $A(\overline{\mathbf{Q}}) \cap B(\overline{\mathbf{Q}})$ is finite. Let $N$ be an integer divisible by the residue characteristics of primes of bad reduction for $J$ (e.g., the conductor of $J$ ). Suppose $p$ is a prime such that

$$
p \nmid 2 \cdot N \cdot \#(J / B)(\mathbf{Q})_{\text {tor }} \cdot \# B(\mathbf{Q})_{\text {tor }} \cdot \prod_{\ell} c_{A, \ell} \cdot c_{B, \ell},
$$

where $c_{A, \ell}=\# \Phi_{A, \ell}\left(\mathbf{F}_{\ell}\right)$ (resp., $c_{B, \ell}$ ) is the Tamagawa number of $A$ (resp., B) at $\ell$. Suppose furthermore that $B[p](\overline{\mathbf{Q}}) \subset A(\overline{\mathbf{Q}})$ as subgroups of $J(\overline{\mathbf{Q}})$. Then there is a natural map

$$
\varphi: B(\mathbf{Q}) / p B(\mathbf{Q}) \rightarrow \operatorname{Vis}_{J}(\amalg(A))
$$

such that $\operatorname{dim}_{\mathbf{F}_{p}} \operatorname{ker}(\varphi) \leq \operatorname{dim}_{\mathbf{Q}} A(\mathbf{Q}) \otimes \mathbf{Q}$.

We return to the situation where $A=A_{f}$ is an optimal quotient of $J_{0}(N)$ attached to a newform. In Proposition 3.15 below we show that $\operatorname{Vis}_{J_{0}(N)}\left(\amalg\left(A^{\vee}\right)\right)$ is annihilated by multiplication by $\operatorname{moddeg}(A)$ (see also CM, p. 19]). We first state a lemma; the outline of the proof was indicated to us by B. Poonen.

Lemma 3.14. Let $A$ be an abelian variety over $k$, where $k$ is a field, and let $\lambda: A \rightarrow A^{\vee}$ be a polarization. Suppose either that $k$ has characteristic 0 or that its characteristic does not divide the degree of $\lambda$. Then there is a finite abelian group $H$ such that $\operatorname{ker}(\lambda) \approx H \times H$ as groups.

Proof. We work in the setting of Section 16 of [Mil86], using the notation used there. Consider the pairing

$$
e^{\lambda}: \operatorname{Ker}(\lambda) \times \operatorname{Ker}(\lambda) \rightarrow \mu_{m} \subseteq \bar{k}^{*},
$$

as in Mil86, p. 135], where $m$ is an integer that kills $\operatorname{Ker}(\lambda)$. We will show that this pairing is nondegenerate.

Suppose $a \in \operatorname{Ker}(\lambda)$ is such that $e^{\lambda}\left(a, a^{\prime}\right)=1$ for all $a^{\prime} \in \operatorname{Ker}(\lambda)$. Let $a^{\prime \prime} \in$ $A^{\vee}[m]$. There exists an isogeny $\lambda^{\prime}: A^{\vee} \rightarrow A$ such that $\lambda^{\prime} \circ \lambda$ is multiplication by $m$ on $A$ and $\lambda \circ \lambda^{\prime}$ is multiplication by $m$ on $A^{\vee}$ (to construct $\lambda^{\prime}$, note that $\lambda^{\prime}$ is the quotient map $\left.A^{\vee} \rightarrow A^{\vee} / \lambda(A[m])\right)$. Pick an element $b \in A(\bar{k})$ such that $\lambda b=a^{\prime \prime}$. Then $m b=\lambda^{\prime}(\lambda b)=\lambda^{\prime}\left(a^{\prime \prime}\right)$. So $\bar{e}_{m}\left(a, a^{\prime \prime}\right)=\bar{e}_{m}(a, \lambda b)=e^{\lambda}\left(a, \lambda^{\prime} a^{\prime \prime}\right)=0$ (note that $\lambda\left(\lambda^{\prime} a^{\prime \prime}\right)=m a^{\prime \prime}=0$, so that $\left.\lambda^{\prime} a^{\prime \prime} \in \operatorname{Ker}(\lambda)\right)$. This is true for all $a^{\prime \prime} \in A^{\vee}[m]$, so the nondegeneracy of $\bar{e}_{m}$ (Mil86, p. 131]) implies that $a=0$.

Similarly, suppose $a^{\prime} \in \operatorname{Ker}(\lambda)$ is such that $e^{\lambda}\left(a, a^{\prime}\right)=1$ for all $a \in \operatorname{Ker}(\lambda)$. Since $e^{\lambda}$ is skew-symmetric ([Mil86, p. 135]), this implies that $e^{\lambda}\left(a^{\prime}, a\right)=1$ for all $a \in \operatorname{Ker}(\lambda)$. Then by the previous paragraph, $a^{\prime}=0$. This finishes the proof of nondegeneracy. 
As mentioned before, the pairing $e^{\lambda}$ is skew-symmetric. It is alternating because it extends to pairings on Tate modules (denoted by $e_{\ell}^{\lambda}$ in Mil86 p. 132]), and the latter take values in a torsion-free group, so there is no distinction between skew-symmetric and alternating.

Now the lemma follows from the fact that if $G$ is a finite abelian group with an alternating nondegenerate pairing, then there is a finite abelian group $H$ such that $G \approx H \times H$ as groups (e.g., see [Del01, Prop. 2]).

Proposition 3.15. Let $m_{A}=\operatorname{moddeg}(A)$. We have

$$
\operatorname{Vis}_{J_{0}(N)}\left(\amalg\left(A^{\vee}\right)\right) \subset \amalg\left(A^{\vee}\right)\left[m_{A}\right] .
$$

Proof. The polarization $\theta_{f}$ (from Section 3.3 ) is the composite map $A^{\vee} \rightarrow J_{0}(N) \rightarrow$ $A$. Let $e_{A}$ be the exponent of the finite group $\operatorname{ker}\left(\theta_{f}\right)$. By Lemma 3.14, multiplication by $m_{A}$ kills $\operatorname{ker}\left(\theta_{f}\right)$, so $e_{A} \mid m_{A}$. Also $\theta_{f}$ factors through multiplication by $e_{A}$, so there is a map $\theta_{f}^{\prime}: A \rightarrow A^{\vee}$ such that $\theta_{f}^{\prime} \circ \theta_{f}$ is multiplication by $e_{A}$. If $\phi$ is a map of abelian varieties (over $\mathbf{Q}$ ), let $\phi_{*}$ denote the corresponding map on Shafarevich-Tate groups. Since $\operatorname{Vis}_{J_{0}(N)}\left(\amalg\left(A^{\vee}\right)\right)$ is contained in $\operatorname{ker}\left(\left(\theta_{f}\right)_{*}\right)$, it is also contained in

$$
\operatorname{ker}\left(\left(\delta^{\prime} \circ \delta\right)_{*}\right)=\amalg\left(A^{\vee}\right)\left[e_{A}\right] \subset \amalg\left(A^{\vee}\right)\left[m_{A}\right] .
$$

Since $\amalg\left(A^{\vee}\right)[n]$ is finite for any $n$, we obtain the following corollary.

Corollary 3.16. $\operatorname{Vis}_{J_{0}(N)}\left(\amalg\left(A^{\vee}\right)\right)$ is finite.

\section{The Quotient $L(A, 1) / \Omega_{A}$}

Fix a newform $f \in S_{2}\left(\Gamma_{0}(N)\right)$, let $I_{f}$ be the annihilator of $f$ in $\mathbf{T}$, and let $A=A_{f}=J_{0}(N) / I_{f} J_{0}(N)$ be the corresponding optimal quotient. Suppose for the rest of this section that $L(A, 1) \neq 0$.

4.1. The Manin constant. When trying to compute the conjectural order of $\amalg(A)$, we try to compute the quotient $L(A, 1) / \Omega_{A}$, but we find that it is easier to compute $c_{A} \cdot L(A, 1) / \Omega_{A}$ where $c_{A}$ is the Manin constant of $A$, which is defined as follows:

Definition 4.1 (Manin constant). The Manin constant of $A$ is

$$
c_{A}=\#\left(\frac{S_{2}\left(\Gamma_{0}(N), \mathbf{Z}\right)\left[I_{f}\right]}{H^{0}\left(\mathcal{A}, \Omega_{\mathcal{A} / \mathbf{Z}}\right)}\right) \in \mathbf{Z}
$$

where we consider $H^{0}\left(\mathcal{A}, \Omega_{\mathcal{A} / \mathbf{Z}}\right)$ as a submodule of $S_{2}\left(\Gamma_{0}(N), \mathbf{Q}\right)$ using

$$
H^{0}\left(\mathcal{A}, \Omega_{\mathcal{A} / \mathbf{Z}}\right) \rightarrow H^{0}\left(\mathcal{J}, \Omega_{\mathcal{J} / \mathbf{Z}}\right)\left[I_{f}\right] \rightarrow H^{0}\left(J, \Omega_{J / \mathbf{Q}}\right)\left[I_{f}\right] \rightarrow S_{2}\left(\Gamma_{0}(N), \mathbf{Q}\right)\left[I_{f}\right],
$$

where $\mathcal{A}$ and $\mathcal{J}$ are the Néron models of $A$ and $J$, respectively. (See [AS04 for a discussion of why the image of $H^{0}\left(\mathcal{A}, \Omega_{\mathcal{A} / \mathbf{Z}}\right)$ is contained in $S_{2}\left(\Gamma_{0}(N), \mathbf{Z}\right)$.)

Theorem 4.2. If $\ell \mid c_{A}$ is a prime, then $\ell^{2} \mid 4 N$.

Proof. Mazur proved this when $\operatorname{dim} A=1$ in [Maz78, §4], and we generalized his proof in [AS04].

When $\operatorname{dim} A=1$, Edixhoven Edi91] obtained strong results towards the folklore conjecture that $c_{A}=1$, and when $A$ has arbitrary dimension, the authors have made the following conjecture (see AS04 for evidence):

Conjecture 4.3. $c_{A}=1$. 
4.2. A formula for $L(A, 1) / \Omega_{A}$. If $L$ and $M$ are lattices in a real vector space $V$, then the lattice index $[L: M]$ is the absolute value of the determinant of a linear transformation of $V$ taking $L$ onto $M$. The lattice index satisfies the usual properties suggested by the notation, e.g., $[L: M] \cdot[M: N]=[L: N]$.

The real volume $\Omega_{A}$ is defined as follows. If $L^{*}$ is a lattice in the cotangent space

$$
T^{*}=H^{0}\left(A_{\mathbf{R}}, \Omega_{A_{\mathbf{R}}}\right)=S_{2}\left(\Gamma_{0}(N), \mathbf{R}\right)\left[I_{f}\right]
$$

of $A_{\mathbf{R}}$, then $L^{*}$ determines a lattice $L=\operatorname{Hom}\left(L^{*}, \mathbf{Z}\right)$ in the tangent space $T=$ $\operatorname{Hom}\left(T^{*}, \mathbf{R}\right)$ and hence a measure on $T$ by declaring that the quotient $T / L$ has measure 1. The induced measure of $A(\mathbf{R})^{0}=T / H_{1}(A(\mathbf{R}), \mathbf{Z})$ is then

$$
\mu_{L}\left(A(\mathbf{R})^{0}\right)=\left[L: H_{1}(A(\mathbf{R}), \mathbf{Z})\right] .
$$

We also set

$$
\mu_{L}(A(\mathbf{R}))=\mu_{L}\left(A(\mathbf{R})^{0}\right) \cdot c_{\infty},
$$

where $c_{\infty}=\#\left(A(\mathbf{R}) / A(\mathbf{R})^{0}\right)$. Let $\mathcal{A}$ be the Néron model of $A$ (see [BLR90]). The Néron differentials $H^{0}\left(\mathcal{A}, \Omega_{\mathcal{A} / \mathbf{z}}\right)$ define a lattice $\Lambda^{*}$ in $T^{*}$, and we define $\Omega_{A}=$ $\mu_{\Lambda}(A(\mathbf{R}))$.

Lemma 4.4. $H_{1}(A(\mathbf{R}), \mathbf{Z}) \cong H_{1}(A(\mathbf{C}), \mathbf{Z})^{+}$.

Proof. This lemma is well known, but we give a proof for the reader's convenience (which was suggested by H. Lenstra and B. Poonen). We have the commutative diagram

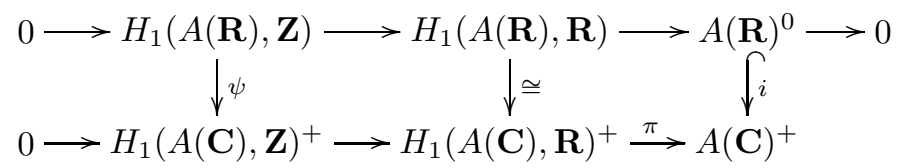

where the upper horizontal sequence is clearly exact, and the lower horizontal sequence is exact because it is the beginning of the long exact sequence of $\mathrm{Gal}(\mathbf{R} / \mathbf{C})$ cohomology that arises from

$$
0 \rightarrow H_{1}(A(\mathbf{C}), \mathbf{Z}) \rightarrow H_{1}(A(\mathbf{C}), \mathbf{R}) \rightarrow A(\mathbf{C}) \rightarrow 0 .
$$

The middle vertical map is an isomorphism because if it were not, then its kernel would be an uncountable set that maps to 0 in $A(\mathbf{R})^{0}$. The snake lemma then yields an exact sequence

$$
0 \rightarrow \operatorname{ker}(\psi) \rightarrow 0 \rightarrow 0 \rightarrow \operatorname{coker}(\psi) \rightarrow 0,
$$

which implies that $\psi$ is an isomorphism.

Let

$$
\Phi: H_{1}\left(X_{0}(N), \mathbf{Q}\right) \rightarrow \operatorname{Hom}\left(S_{2}\left(\Gamma_{0}(N)\right)\left[I_{f}\right], \mathbf{C}\right)
$$

be the map induced by integration, scaled so that

$$
\Phi(\{0, \infty\})(f)=L(f, 1)
$$

(that $\{0, \infty\} \in H_{1}\left(X_{0}(N), \mathbf{Q}\right)$ is the Manin-Drinfeld theorem and that $\int_{0}^{\infty} f$ is a multiple of $L(f, 1)$ follows from the definition of $L(f, s)$ as a Mellin transform). 
Theorem 4.5. Recall that $A$ is an abelian variety attached to a newform $f \in$ $S_{2}\left(\Gamma_{0}(N)\right)$, that $c_{\infty}$ is the number of connected components of $A(\mathbf{R})$, that $c_{A}$ is the Manin constant of $A$, that $\Omega_{A}$ is the Néron canonical volume of $A(\mathbf{R})$, and that $\Phi$ is the period mapping on the homology induced by integrating homology classes on $X_{0}(N)$ against the $\mathbf{C}$-vector space spanned by the $\operatorname{Gal}(\overline{\mathbf{Q}} / \mathbf{Q})$-conjugates of $f$. Then we have the following equation:

$$
c_{\infty} \cdot c_{A} \cdot \frac{L(A, 1)}{\Omega_{A}}=\left[\Phi\left(H_{1}\left(X_{0}(N), \mathbf{Z}\right)\right)^{+}: \Phi(\mathbf{T}\{0, \infty\})\right] \in \mathbf{Q},
$$

where the lattice index on the right-hand side should be interpreted as 0 if $\Phi(\mathbf{T}\{0, \infty\})$ has rank less than the dimension of $A$.

Proof. It is easier to compute with $\tilde{\Lambda}^{*}=S_{2}\left(\Gamma_{0}(N), \mathbf{Z}\right)\left[I_{f}\right]$ than with $\Lambda^{*}$, so let $\tilde{\Omega}_{A}=\mu_{\tilde{\Lambda}}(A(\mathbf{R}))$. Note that $\tilde{\Omega}_{A} \cdot c_{A}=\Omega_{A}$, where $c_{A}$ is the Manin constant. By Lemma 4.4 and Section 2.2.

$$
\begin{aligned}
\tilde{\Omega}_{A} & =c_{\infty} \cdot\left[\tilde{\Lambda}: H_{1}(A(\mathbf{R}), \mathbf{Z})\right] \\
& =c_{\infty} \cdot\left[\operatorname{Hom}\left(S_{2}\left(\Gamma_{0}(N), \mathbf{Z}\right)\left[I_{f}\right], \mathbf{Z}\right): \Phi\left(H_{1}\left(X_{0}(N), \mathbf{Z}\right)\right)^{+}\right] .
\end{aligned}
$$

For any ring $R$ the pairing

$$
\mathbf{T}_{R} \times S_{2}\left(\Gamma_{0}(N), R\right) \rightarrow R
$$

given by $\left\langle T_{n}, f\right\rangle=a_{1}\left(T_{n} f\right)$ is perfect, so $\left(\mathbf{T} / I_{f}\right) \otimes R \cong \operatorname{Hom}\left(S_{2}\left(\Gamma_{0}(N), R\right)\left[I_{f}\right], R\right)$. Using this pairing, we may view $\Phi$ as a map

$$
\Phi: H_{1}\left(X_{0}(N), \mathbf{Q}\right) \rightarrow\left(\mathbf{T} / I_{f}\right) \otimes \mathbf{C},
$$

so that

$$
\tilde{\Omega}_{A}=c_{\infty} \cdot\left[\mathbf{T} / I_{f}: \Phi\left(H_{1}\left(X_{0}(N), \mathbf{Z}\right)\right)^{+}\right] .
$$

Note that $\left(\mathbf{T} / I_{f}\right) \otimes \mathbf{C}$ is isomorphic as a ring to a product of copies of $\mathbf{C}$, with one copy corresponding to each Galois conjugate $f^{(i)}$ of $f$. Let $\pi_{i} \in\left(\mathbf{T} / I_{f}\right) \otimes \mathbf{C}$ be the projector onto the subspace of $\left(\mathbf{T} / I_{f}\right) \otimes \mathbf{C}$ corresponding to $f^{(i)}$. Then $\Phi(\{0, \infty\}) \cdot \pi_{i}=L\left(f^{(i)}, 1\right) \cdot \pi_{i}$. Since the $\pi_{i}$ form a basis for the complex vector space $\left(\mathbf{T} / I_{f}\right) \otimes \mathbf{C}$, we see that

$$
\operatorname{det}(\Phi(\{0, \infty\}))=\prod_{i} L\left(f^{(i)}, 1\right)=L(A, 1) .
$$

Letting $H=H_{1}\left(X_{0}(N), \mathbf{Z}\right)$, we have

$$
\begin{aligned}
{\left[\Phi(H)^{+}: \Phi(\mathbf{T}\{0, \infty\})\right] } & =\left[\Phi(H)^{+}:\left(\mathbf{T} / I_{f}\right) \cdot \Phi(\{0, \infty\})\right] \\
& =\left[\Phi(H)^{+}: \mathbf{T} / I_{f}\right] \cdot\left[\mathbf{T} / I_{f}: \mathbf{T} / I_{f} \cdot \Phi(\{0, \infty\})\right] \\
& =\frac{c_{\infty}}{\tilde{\Omega}_{A}} \cdot \operatorname{det}(\Phi(\{0, \infty\})) \\
& =\frac{c_{\infty} c_{A}}{\Omega_{A}} \cdot L(A, 1),
\end{aligned}
$$

which proves the theorem.

Theorem 4.5 was inspired by the case when $A$ is an elliptic curve (see Cre97. $\S[I .2 .8]$ ) or the winding quotient of $J_{0}(p)$ (see Aga99), and it generalizes to forms of weight $>2$ (see [Ste00]).

Theorem [4.5 is true with $\Phi$ replaced by any linear map with the same kernel as $\Phi$. One way to find such a linear map with image in a $\mathbf{Q}$-vector space is to 
compute a basis $\varphi_{1}, \ldots, \varphi_{d}$ for $\operatorname{Hom}\left(H_{1}\left(X_{0}(N), \mathbf{Q}\right), \mathbf{Q}\right)\left[I_{f}\right]$ and let $\Phi=\varphi_{1} \times \cdots \times$ $\varphi_{d}$. Also, since $H_{1}\left(X_{0}(N), \mathbf{Z}\right)^{+}$and $\mathbf{T}\{0, \infty\}$ are contained in $H_{1}\left(X_{0}(N), \mathbf{Q}\right)^{+}$, Theorem 4.5 implies that $L(A, 1) / \Omega_{A} \in \mathbf{Q}$, a fact well known to the experts (see [Gro94, Prop. 2.7] for the statement, but without proof).

4.3. The denominator of $L(A, 1) / \Omega_{A}$. In this section, we prove a result about the denominator of the rational number $L(A, 1) / \Omega_{A}$ and we compare it to what is predicted by the Birch and Swinnerton-Dyer conjecture.

Proposition 4.6. Let $z$ be the point in $J_{0}(N)(\mathbf{Q})$ defined by the degree 0 divisor $(0)-(\infty)$ on $X_{0}(N)$, and let $n=n_{f}$ be the order of the image of $z$ in $A(\mathbf{Q})$. Then the denominator of $c_{\infty} \cdot c_{A} \cdot L(A, 1) / \Omega_{A}$ divides $n$.

Proof. Let $x$ be the image of $z$ in $A(\mathbf{Q})$, and let $I=\operatorname{Ann}_{\mathbf{T}}(x)$ be the ideal of elements of $\mathbf{T}$ that annihilate $x$. Since $f$ is a newform, the Hecke operators $T_{p}$, for $p \mid N$, act as 0 or \pm 1 on $A(\mathbf{Q})$ (see, e.g., DI95 $\S 6]$ ). If $p \nmid N$, then a standard calculation (see, e.g., [re97, §2.8]) shows that $T_{p}(x)=(p+1) x$.

Let $C$ be the cyclic subgroup of $A(\mathbf{Q})$ of order $n$ generated by $x$. Consider the map $\mathbf{T} \rightarrow C$ given by $T_{p} \mapsto T_{p}(x)$. The kernel of this map is $I$, and the map is surjective because its image is an additive group that contains $x$, and $C$ is the smallest such group. Thus the map induces an isomorphism $\mathbf{T} / I \stackrel{\cong}{\rightrightarrows} C$.

Conjecture 2.2 predicts that

$$
\# A(\mathbf{Q}) \cdot \# A^{\vee}(\mathbf{Q}) \cdot \frac{L(A, 1)}{\Omega_{A}}=\# \amalg(A) \cdot \prod c_{p} \in \mathbf{Z},
$$

and since $n \mid \# A(\mathbf{Q})$, Proposition 4.6 implies that

$$
c_{\infty} \cdot c_{A} \cdot \# A(\mathbf{Q}) \cdot \frac{L(A, 1)}{\Omega_{A}} \in \mathbf{Z} .
$$

Since $c_{\infty}$ is a power of 2 and $c_{A}$ is conjecturally 1 (if $N$ is prime, then by Theorem 4.2 it is a power of 2), Proposition 4.6]provides theoretical evidence for Conjecture 2.2 and also reflects a surprising amount of cancellation between $\prod c_{p}$ and $\# A^{\vee}(\mathbf{Q})$.

\section{Results And CONCLUSIONS}

We computed all 19608 abelian varieties $A=A_{f}$ attached to newforms of level $N \leq 2333$. Interesting data about some of these abelian varieties is summarized in Tables 14, which use the notation described in this section.

Suppose that $A$ is one of the 10360 of these for which $L(A, 1) \neq 0$, so Conjecture 2.2 asserts that $\amalg(A)$ has order

$$
\# \amalg_{?}=\frac{L(A, 1)}{\tilde{\Omega}_{A} \cdot c_{A}} \cdot \frac{\# A(\mathbf{Q})_{\text {tor }} \cdot \# A^{\vee}(\mathbf{Q})_{\text {tor }}}{\prod_{p \mid N} c_{p}} .
$$

(See Section 4.2 for the definition of $\tilde{\Omega}_{A}$ and $c_{A}$.)

For any rational number $x$, let $x^{\text {odd }}$ be the odd part of $x$. If $a$ and $b$ are rational numbers with $a \neq 0$, we say that $a \mid b$ if $b / a$ is an integer. 
TABLE 1. Visibility of nontrivial odd parts of Shafarevich-Tate groups

\begin{tabular}{|c|c|c|c|c|c|c|c|c|}
\hline$A$ & $\operatorname{dim}$ & $S_{l}$ & $S_{u}$ & moddeg $(A)^{\text {odd }}$ & $B$ & $\operatorname{dim}$ & $A^{\vee} \cap \tilde{B}^{\vee}$ & Vis \\
\hline $389 \mathrm{E} *$ & 20 & $5^{2}$ & $=$ & 5 & 389A & 1 & {$\left[20^{2}\right]$} & $5^{2}$ \\
\hline $433 \mathrm{D} *$ & 16 & $7^{2}$ & $=$ & $7 \cdot 111$ & $433 \mathrm{~A}$ & 1 & {$\left[14^{2}\right]$} & $7^{2}$ \\
\hline $446 \mathrm{~F} *$ & 8 & $11^{2}$ & $=$ & $11 \cdot 359353$ & 446B & 1 & {$\left[11^{2}\right]$} & $11^{2}$ \\
\hline $551 \mathrm{H}$ & 18 & $3^{2}$ & $=$ & 169 & NONE & & & \\
\hline $563 \mathrm{E} *$ & 31 & $13^{2}$ & $=$ & 13 & $563 \mathrm{~A}$ & 1 & {$\left[26^{2}\right]$} & $13^{2}$ \\
\hline 571D* & 2 & $3^{2}$ & $=$ & $3^{2} \cdot 127$ & 571B & 1 & {$\left[3^{2}\right]$} & $3^{2}$ \\
\hline $655 \mathrm{D} *$ & 13 & $3^{4}$ & $=$ & $3^{2} \cdot 9799079$ & $655 \mathrm{~A}$ & 1 & {$\left[36^{2}\right]$} & $3^{4}$ \\
\hline $681 \mathrm{~B}$ & 1 & $3^{2}$ & $=$ & $3 \cdot 125$ & $681 \mathrm{C}$ & 1 & {$\left[3^{2}\right]$} & - \\
\hline $707 \mathrm{G} *$ & 15 & $13^{2}$ & $=$ & $13 \cdot 800077$ & $707 \mathrm{~A}$ & 1 & {$\left[13^{2}\right]$} & $13^{2}$ \\
\hline 709C $*$ & 30 & $11^{2}$ & $=$ & 11 & 709A & 1 & {$\left[22^{2}\right]$} & $11^{2}$ \\
\hline $718 \mathrm{~F} *$ & 7 & $7^{2}$ & $=$ & $7 \cdot 5371523$ & $718 \mathrm{~B}$ & 1 & {$\left[7^{2}\right]$} & $7^{2}$ \\
\hline $767 \mathrm{~F}$ & 23 & $3^{2}$ & $=$ & 1 & NONE & & & \\
\hline $794 \mathrm{G}$ & 12 & $11^{2}$ & $=$ & $11 \cdot 34986189$ & $794 \mathrm{~A}$ & 1 & {$\left[11^{2}\right]$} & - \\
\hline $817 \mathrm{E}$ & 15 & $7^{2}$ & $=$ & $7 \cdot 79$ & $817 \mathrm{~A}$ & 1 & {$\left[7^{2}\right]$} & - \\
\hline 959D & 24 & $3^{2}$ & $=$ & 583673 & NONE & & & \\
\hline $997 \mathrm{H} *$ & 42 & $3^{4}$ & $=$ & $3^{2}$ & $997 \mathrm{~B}$ & 1 & {$\left[12^{2}\right]$} & $3^{2}$ \\
\hline & & & & & $997 \mathrm{C}$ & & {$\left[24^{2}\right]$} & $3^{2}$ \\
\hline $1001 \mathrm{~F}$ & 3 & $3^{2}$ & $=$ & $3^{2} \cdot 1269$ & $1001 \mathrm{C}$ & 1 & {$\left[3^{2}\right]$} & - \\
\hline & & & & & $91 \mathrm{~A}$ & 1 & {$\left[3^{2}\right]$} & - \\
\hline $1001 \mathrm{~L}$ & 7 & $7^{2}$ & $=$ & $7 \cdot 2029789$ & $1001 \mathrm{C}$ & 1 & {$\left[7^{2}\right]$} & - \\
\hline $1041 \mathrm{E}$ & 4 & $5^{2}$ & $=$ & $5^{2} \cdot 13589$ & 1041B & 2 & {$\left[5^{2}\right]$} & - \\
\hline $1041 \mathrm{~J}$ & 13 & $5^{4}$ & $=$ & $5^{3} \cdot 21120929983$ & 1041B & 2 & {$\left[5^{4}\right]$} & - \\
\hline $1058 D$ & 1 & $5^{2}$ & $=$ & $5 \cdot 483$ & $1058 \mathrm{C}$ & 1 & {$\left[5^{2}\right]$} & - \\
\hline 1061D & 46 & $151^{2}$ & $=$ & $151 \cdot 10919$ & 1061B & 2 & {$\left[2^{2} 302^{2}\right]$} & - \\
\hline $1070 \mathrm{M}$ & 7 & $3 \cdot 5^{2}$ & $3^{2} \cdot 5^{2}$ & $3 \cdot 5 \cdot 1720261$ & $1070 \mathrm{~A}$ & 1 & {$\left[15^{2}\right]$} & - \\
\hline $1077 \mathrm{~J}$ & 15 & $3^{4}$ & $=$ & $3^{2} \cdot 1227767047943$ & $1077 \mathrm{~A}$ & 1 & {$\left[9^{2}\right]$} & - \\
\hline $1091 \mathrm{C}$ & 62 & $7^{2}$ & $=$ & 1 & NONE & & & \\
\hline $1094 \mathrm{~F} *$ & 13 & $11^{2}$ & $=$ & $11^{2} \cdot 172446773$ & $1094 \mathrm{~A}$ & 1 & {$\left[11^{2}\right]$} & $11^{2}$ \\
\hline $1102 \mathrm{~K}$ & 4 & $3^{2}$ & $=$ & $3^{2} \cdot 31009$ & $1102 \mathrm{~A}$ & 1 & {$\left[3^{2}\right]$} & - \\
\hline $1126 \mathrm{~F} *$ & 11 & $11^{2}$ & $=$ & $11 \cdot 13990352759$ & $1126 \mathrm{~A}$ & 1 & {$\left[11^{2}\right]$} & $11^{2}$ \\
\hline $1137 \mathrm{C}$ & 14 & $3^{4}$ & $=$ & $3^{2} \cdot 64082807$ & $1137 \mathrm{~A}$ & 1 & {$\left[9^{2}\right]$} & - \\
\hline $1141 \mathrm{I}$ & 22 & $7^{2}$ & $=$ & $7 \cdot 528921$ & $1141 \mathrm{~A}$ & 1 & {$\left[14^{2}\right]$} & - \\
\hline $1147 \mathrm{H}$ & 23 & $5^{2}$ & $=$ & $5 \cdot 729$ & $1147 \mathrm{~A}$ & 1 & {$\left[10^{2}\right]$} & - \\
\hline $1171 \mathrm{D} *$ & 53 & $11^{2}$ & $=$ & $11 \cdot 81$ & $1171 \mathrm{~A}$ & 1 & {$\left[44^{2}\right]$} & $11^{2}$ \\
\hline $1246 \mathrm{~B}$ & 1 & $5^{2}$ & $=$ & $5 \cdot 81$ & $1246 \mathrm{C}$ & 1 & {$\left[5^{2}\right]$} & - \\
\hline 1247D & 32 & $3^{2}$ & $=$ & $3^{2} \cdot 2399$ & 43A & 1 & {$\left[36^{2}\right]$} & - \\
\hline $1283 \mathrm{C}$ & 62 & $5^{2}$ & $=$ & $5 \cdot 2419$ & NONE & & & \\
\hline $1337 \mathrm{E}$ & 33 & $3^{2}$ & $=$ & 71 & NONE & & & \\
\hline 1339G & 30 & $3^{2}$ & $=$ & 5776049 & NONE & & & \\
\hline $1355 \mathrm{E}$ & 28 & 3 & $3^{2}$ & $3^{2} \cdot 2224523985405$ & NONE & & & \\
\hline $1363 \mathrm{~F}$ & 25 & $31^{2}$ & $=$ & $31 \cdot 34889$ & 1363B & 2 & {$\left[2^{2} 62^{2}\right]$} & - \\
\hline 1429B & 64 & $5^{2}$ & $=$ & 1 & NONE & & & \\
\hline 1443G & 5 & $7^{2}$ & $=$ & $7^{2} \cdot 18525$ & $1443 \mathrm{C}$ & 1 & {$\left[7^{1} 14^{1}\right]$} & - \\
\hline $1446 \mathrm{~N}$ & 7 & $3^{2}$ & $=$ & $3 \cdot 17459029$ & 1446A & 1 & {$\left[12^{2}\right]$} & - \\
\hline
\end{tabular}


TABLE 2. Visibility of nontrivial odd parts of Shafarevich-Tate groups

\begin{tabular}{|c|c|c|c|c|c|c|c|c|}
\hline$A$ & $\operatorname{dim}$ & $S_{l}$ & $S_{u}$ & moddeg $(A)^{\text {odd }}$ & $B$ & $\operatorname{dim}$ & $A^{\vee} \cap \tilde{B}^{\vee}$ & Vis \\
\hline $1466 \mathrm{H} *$ & 23 & $13^{2}$ & $=$ & $13 \cdot 25631993723$ & 1466B & 1 & {$\left[26^{2}\right]$} & $13^{2}$ \\
\hline $1477 \mathrm{C} *$ & 24 & $13^{2}$ & $=$ & $13 \cdot 57037637$ & $1477 \mathrm{~A}$ & 1 & {$\left[13^{2}\right]$} & $13^{2}$ \\
\hline $1481 \mathrm{C}$ & 71 & $13^{2}$ & $=$ & 70825 & NONE & & & \\
\hline $1483 \mathrm{D} *$ & 67 & $3^{2} \cdot 5^{2}$ & $=$ & $3 \cdot 5$ & $1483 \mathrm{~A}$ & 1 & {$\left[60^{2}\right]$} & $3^{2} \cdot 5^{2}$ \\
\hline $1513 F$ & 31 & 3 & $3^{4}$ & $3 \cdot 759709$ & NONE & & & \\
\hline $1529 \mathrm{D}$ & 36 & $5^{2}$ & $=$ & 535641763 & NONE & & & \\
\hline 1531D & 73 & 3 & $3^{2}$ & 3 & $1531 \mathrm{~A}$ & 1 & {$\left[48^{2}\right]$} & - \\
\hline $1534 \mathrm{~J}$ & 6 & 3 & $3^{2}$ & $3^{2} \cdot 635931$ & $1534 \mathrm{~B}$ & 1 & {$\left[6^{2}\right]$} & - \\
\hline $1551 \mathrm{G}$ & 13 & $3^{2}$ & $=$ & $3 \cdot 110659885$ & $141 \mathrm{~A}$ & 1 & {$\left[15^{2}\right]$} & - \\
\hline 1559B & 90 & $11^{2}$ & $=$ & 1 & NONE & & & \\
\hline $1567 \mathrm{D}$ & 69 & $7^{2} \cdot 41^{2}$ & $=$ & $7 \cdot 41$ & $1567 \mathrm{~B}$ & 3 & {$\left[4^{4} 1148^{2}\right]$} & - \\
\hline $1570 J *$ & 6 & $11^{2}$ & $=$ & $11 \cdot 228651397$ & 1570B & 1 & {$\left[11^{2}\right]$} & $11^{2}$ \\
\hline $1577 \mathrm{E}$ & 36 & 3 & $3^{2}$ & $3^{2} \cdot 15$ & $83 \mathrm{~A}$ & & {$\left[6^{2}\right]$} & - \\
\hline $1589 \mathrm{D}$ & 35 & $3^{2}$ & $=$ & 6005292627343 & NONE & & & \\
\hline $1591 \mathrm{~F} *$ & 35 & $31^{2}$ & $=$ & $31 \cdot 2401$ & $1591 \mathrm{~A}$ & 1 & {$\left[31^{2}\right]$} & $31^{2}$ \\
\hline $1594 \mathrm{~J}$ & 17 & $3^{2}$ & $=$ & $3 \cdot 259338050025131$ & $1594 \mathrm{~A}$ & 1 & {$\left[12^{2}\right]$} & - \\
\hline $1613 \mathrm{D} *$ & 75 & $5^{2}$ & $=$ & $5 \cdot 19$ & $1613 \mathrm{~A}$ & 1 & {$\left[20^{2}\right]$} & $5^{2}$ \\
\hline $1615 \mathrm{~J}$ & 13 & $3^{4}$ & $=$ & $3^{2} \cdot 13317421$ & 1615A & 1 & {$\left[9^{1} 18^{1}\right]$} & - \\
\hline $1621 \mathrm{C} *$ & 70 & $17^{2}$ & $=$ & 17 & $1621 \mathrm{~A}$ & 1 & {$\left[34^{2}\right]$} & $17^{2}$ \\
\hline $1627 \mathrm{C} *$ & 73 & $3^{4}$ & $=$ & $3^{2}$ & $1627 \mathrm{~A}$ & 1 & {$\left[36^{2}\right]$} & $3^{4}$ \\
\hline $1631 \mathrm{C}$ & 37 & $5^{2}$ & $=$ & 6354841131 & NONE & & & \\
\hline $1633 \mathrm{D}$ & 27 & $3^{6} \cdot 7^{2}$ & $=$ & $3^{5} \cdot 7 \cdot 31375$ & 1633A & 3 & {$\left[6^{4} 42^{2}\right]$} & - \\
\hline $1634 \mathrm{~K}$ & 12 & $3^{2}$ & $=$ & $3 \cdot 3311565989$ & $817 \mathrm{~A}$ & 1 & {$\left[3^{2}\right]$} & - \\
\hline $1639 \mathrm{G} *$ & 34 & $17^{2}$ & $=$ & $17 \cdot 82355$ & 1639B & 1 & {$\left[34^{2}\right]$} & $17^{2}$ \\
\hline $1641 \mathrm{~J} *$ & 24 & $23^{2}$ & $=$ & $23 \cdot 1491344147471$ & 1641B & 1 & {$\left[23^{2}\right]$} & $23^{2}$ \\
\hline $1642 \mathrm{D} *$ & 14 & $7^{2}$ & $=$ & $7 \cdot 123398360851$ & $1642 \mathrm{~A}$ & 1 & {$\left[7^{2}\right]$} & $7^{2}$ \\
\hline $1662 \mathrm{~K}$ & 7 & $11^{2}$ & $=$ & $11 \cdot 16610917393$ & $1662 \mathrm{~A}$ & 1 & {$\left[11^{2}\right]$} & - \\
\hline $1664 \mathrm{~K}$ & 1 & $5^{2}$ & $=$ & $5 \cdot 7$ & $1664 \mathrm{~N}$ & 1 & {$\left[5^{2}\right]$} & - \\
\hline $1679 \mathrm{C}$ & 45 & $11^{2}$ & $=$ & 6489 & NONE & & & \\
\hline $1689 \mathrm{E}$ & 28 & $3^{2}$ & $=$ & $\cdot 172707180029157365$ & $563 \mathrm{~A}$ & 1 & {$\left[3^{2}\right]$} & - \\
\hline $1693 \mathrm{C}$ & 72 & $1301^{2}$ & $=$ & 1301 & 1693A & 3 & {$\left[2^{4} 2602^{2}\right]$} & - \\
\hline $1717 \mathrm{H} *$ & 34 & $13^{2}$ & $=$ & $13 \cdot 345$ & 1717B & 1 & {$\left[26^{2}\right]$} & $13^{2}$ \\
\hline $1727 \mathrm{E}$ & 39 & $3^{2}$ & $=$ & 118242943 & NONE & & & \\
\hline $1739 \mathrm{~F}$ & 43 & $659^{2}$ & $=$ & $659 \cdot 151291281$ & $1739 \mathrm{C}$ & 2 & {$\left[2^{2} 1318^{2}\right]$} & - \\
\hline $1745 \mathrm{~K}$ & 33 & $5^{2}$ & $=$ & $5 \cdot 1971380677489$ & $1745 \mathrm{D}$ & 1 & {$\left[20^{2}\right]$} & - \\
\hline $1751 \mathrm{C}$ & 45 & $5^{2}$ & $=$ & $5 \cdot 707$ & $103 \mathrm{~A}$ & 2 & {$\left[505^{2}\right]$} & - \\
\hline $1781 \mathrm{D}$ & 44 & $3^{2}$ & $=$ & 61541 & NONE & & & \\
\hline $1793 \mathrm{G} *$ & 36 & $23^{2}$ & $=$ & $23 \cdot 8846589$ & 1793B & 1 & {$\left[23^{2}\right]$} & $23^{2}$ \\
\hline 1799D & 44 & $5^{2}$ & $=$ & 201449 & NONE & & & \\
\hline 1811D & 98 & $31^{2}$ & $=$ & 1 & NONE & & & \\
\hline $1829 \mathrm{E}$ & 44 & $13^{2}$ & $=$ & 3595 & NONE & & & \\
\hline $1843 F$ & 40 & $3^{2}$ & $=$ & 8389 & NONE & & & \\
\hline $1847 \mathrm{~B}$ & 98 & $3^{6}$ & $=$ & 1 & NONE & & & \\
\hline $1871 \mathrm{C}$ & 98 & $19^{2}$ & $=$ & 14699 & NONE & & & \\
\hline
\end{tabular}


TABLE 3. Visibility of nontrivial odd parts of Shafarevich-Tate groups

\begin{tabular}{|c|c|c|c|c|c|c|c|c|}
\hline$A$ & $\operatorname{dim}$ & $S_{l}$ & $S_{u}$ & moddeg $(A)^{\text {odd }}$ & $B$ & $\operatorname{dim}$ & $A^{\vee} \cap \tilde{B}^{\vee}$ & Vis \\
\hline $1877 \mathrm{~B}$ & 86 & $7^{2}$ & $=$ & 1 & NONE & & & \\
\hline $1887 \mathrm{~J}$ & 12 & $5^{2}$ & $=$ & $5 \cdot 10825598693$ & $1887 \mathrm{~A}$ & & {$\left[20^{2}\right]$} & - \\
\hline $1891 \mathrm{H}$ & 40 & $7^{4}$ & $=$ & $7^{2} \cdot 44082137$ & $1891 \mathrm{C}$ & & {$\left[4^{2} 196^{2}\right]$} & - \\
\hline $1907 \mathrm{D} *$ & 90 & $7^{2}$ & $=$ & $7 \cdot 165$ & $1907 \mathrm{~A}$ & 1 & {$\left[56^{2}\right]$} & $7^{2}$ \\
\hline $1909 \mathrm{D} *$ & 38 & $3^{4}$ & $=$ & $3^{2} \cdot 9317$ & 1909A & & {$\left[18^{2}\right]$} & $3^{4}$ \\
\hline 1913B $*$ & 1 & $3^{2}$ & $=$ & $3 \cdot 103$ & 1913A & & {$\left[3^{2}\right]$} & $3^{2}$ \\
\hline $1913 E$ & 84 & $5^{4} \cdot 61^{2}$ & $=$ & $5^{2} \cdot 61 \cdot 103$ & $1913 \mathrm{~A}$ & & {$\left[10^{2}\right]$} & - \\
\hline & & & & & $1913 \mathrm{C}$ & & {$\left[2^{2} 610^{2}\right]$} & - \\
\hline 1919D & 52 & $23^{2}$ & $=$ & 675 & NONE & & & \\
\hline $1927 \mathrm{E}$ & 45 & $3^{2}$ & $3^{4}$ & 52667 & NONE & & & \\
\hline $1933 \mathrm{C}$ & 83 & $3^{2} \cdot 7$ & $3^{2} \cdot 7^{2}$ & $3 \cdot 7$ & $1933 \mathrm{~A}$ & 1 & {$\left[42^{2}\right]$} & $3^{2}$ \\
\hline $1943 \mathrm{E}$ & 46 & $13^{2}$ & $=$ & 62931125 & NONE & & & \\
\hline $1945 \mathrm{E} *$ & 34 & $3^{2}$ & $=$ & $3 \cdot 571255479184807$ & $389 \mathrm{~A}$ & & {$\left[3^{2}\right]$} & $3^{2}$ \\
\hline $1957 \mathrm{E} *$ & 37 & $7^{2} \cdot 11^{2}$ & $=$ & $7 \cdot 11 \cdot 3481$ & 1957A & & {$\left[22^{2}\right]$} & $11^{2}$ \\
\hline $1979 \mathrm{C}$ & 104 & $19^{2}$ & $=$ & 55 & 1957B & & {$\left[14^{2}\right]$} & $7^{2}$ \\
\hline $1991 \mathrm{C}$ & 49 & $7^{2}$ & $=$ & 1634403663 & NONE & & & \\
\hline $1994 \mathrm{D}$ & 26 & 3 & $3^{2}$ & $3^{2} \cdot 46197281414642501$ & $997 \mathrm{~B}$ & 1 & {$\left[3^{2}\right]$} & - \\
\hline $1997 \mathrm{C}$ & 93 & $17^{2}$ & $=$ & 1 & NONE & & & \\
\hline 2001L & 11 & $3^{2}$ & $=$ & $3^{2} \cdot 44513447$ & NONE & & & \\
\hline $2006 \mathrm{E}$ & 1 & $3^{2}$ & $=$ & $3 \cdot 805$ & $2006 \mathrm{D}$ & 1 & {$\left[3^{2}\right]$} & - \\
\hline 2014L & 12 & $3^{2}$ & $=$ & $3^{2} \cdot 126381129003$ & 106A & 1 & {$\left[9^{2}\right]$} & - \\
\hline $2021 E$ & 50 & $5^{6}$ & $=$ & $5^{2} \cdot 729$ & 2021A & & {$\left[100^{2}\right]$} & $5^{4}$ \\
\hline $2027 \mathrm{C} *$ & 94 & $29^{2}$ & $=$ & 29 & 2027 A & 1 & {$\left[58^{2}\right]$} & $29^{2}$ \\
\hline $2029 \mathrm{C}$ & 90 & $5^{2} \cdot 269^{2}$ & $=$ & $5 \cdot 269$ & 2029A & & {$\left[2^{2} 2690^{2}\right]$} & - \\
\hline 2031H* & 36 & $11^{2}$ & $=$ & $11 \cdot 1014875952355$ & $2031 \mathrm{C}$ & 1 & {$\left[44^{2}\right]$} & $11^{2}$ \\
\hline $2035 \mathrm{~K}$ & 16 & $11^{2}$ & $=$ & $11 \cdot 218702421$ & $2035 \mathrm{C}$ & 1 & {$\left[11^{1} 22^{1}\right]$} & - \\
\hline 2038F & 25 & 5 & $5^{2}$ & $5^{2} \cdot 92198576587$ & 2038A & 1 & {$\left[20^{2}\right]$} & - \\
\hline & & & & & 1019B & & {$\left[5^{2}\right]$} & - \\
\hline 2039F & 99 & $3^{4} \cdot 5^{2}$ & $=$ & 13741381043009 & NONE & & & \\
\hline $2041 C$ & 43 & $3^{4}$ & $=$ & 61889617 & NONE & & & \\
\hline 2045 I & 39 & $3^{4}$ & $=$ & $3^{3} \cdot 3123399893$ & $2045 \mathrm{C}$ & 1 & {$\left[18^{2}\right]$} & - \\
\hline & & & & & $409 \mathrm{~A}$ & & {$\left[9370199679^{2}\right]$} & - \\
\hline 2049D & 31 & $3^{2}$ & $=$ & 29174705448000469937 & NONE & & & \\
\hline 2051D & 45 & $7^{2}$ & $=$ & $7 \cdot 674652424406369$ & 2051A & 1 & {$\left[56^{2}\right]$} & - \\
\hline $2059 \mathrm{E}$ & 45 & $5 \cdot 7^{2}$ & $5^{2} \cdot 7^{2}$ & $5^{2} \cdot 7 \cdot 167359757$ & 2059A & 1 & {$\left[70^{2}\right]$} & - \\
\hline $2063 \mathrm{C}$ & 106 & $13^{2}$ & $=$ & 8479 & NONE & & & \\
\hline $2071 F$ & 48 & $13^{2}$ & $=$ & 36348745 & NONE & & & \\
\hline 2099B & 106 & $3^{2}$ & $=$ & 1 & NONE & & & \\
\hline 2101F & 46 & $5^{2}$ & $=$ & $5 \cdot 11521429$ & $191 \mathrm{~A}$ & 2 & {$\left[155^{2}\right]$} & - \\
\hline $2103 E$ & 37 & $3^{2} \cdot 11^{2}$ & $=3$ & $3^{2} \cdot 11 \cdot 874412923071571792611$ & 2103B & & {$\left[33^{2}\right]$} & $11^{2}$ \\
\hline 2111B & 112 & $211^{2}$ & $=$ & 1 & NONE & & & \\
\hline 2113B & 91 & $7^{2}$ & $=$ & 1 & NONE & & & \\
\hline $2117 \mathrm{E} *$ & 45 & $19^{2}$ & $=$ & $19 \cdot 1078389$ & 2117 A & 1 & {$\left[38^{2}\right]$} & $19^{2}$ \\
\hline
\end{tabular}


TABLE 4. Visibility of nontrivial odd parts of Shafarevich-Tate groups

\begin{tabular}{|c|c|c|c|c|c|c|c|c|}
\hline$A$ & $\operatorname{dim}$ & $S_{l}$ & $S_{u}$ & moddeg $(A)^{\text {odd }}$ & $B$ & $\operatorname{dim}$ & $A^{\vee} \cap \tilde{B}^{\vee}$ & Vis \\
\hline $2119 \mathrm{C}$ & 48 & $7^{2}$ & $=$ & 89746579 & NONE & & \multirow{3}{*}[3^{2}]{} & \multirow{3}{*}{-} \\
\hline 2127D & 34 & $3^{2}$ & $=$ & $3 \cdot 18740561792121901$ & 709A & 1 & & \\
\hline 2129B & 102 & $3^{2}$ & $=$ & 1 & NONE & & & \\
\hline $2130 Y$ & 4 & $7^{2}$ & $=$ & $7 \cdot 83927$ & 2130B & 1 & {$\left[14^{2}\right]$} & - \\
\hline 2131B & 101 & $17^{2}$ & $=$ & 1 & NONE & \multirow{4}{*}{1} & \multirow{4}{*}[7^{2}]{} & \multirow{4}{*}{-} \\
\hline $2134 J$ & 11 & $3^{2}$ & $=$ & 1710248025389 & NONE & & & \\
\hline 2146J & 10 & $7^{2}$ & $=$ & $7 \cdot 1672443$ & 2146A & & & \\
\hline 2159E & 57 & $13^{2}$ & $=$ & 31154538351 & NONE & & & \\
\hline 2159D & 56 & $3^{4}$ & $=$ & 233801 & NONE & & & \\
\hline $2161 \mathrm{C}$ & 98 & $23^{2}$ & $=$ & 1 & NONE & & & \\
\hline $2162 \mathrm{H}$ & 14 & 3 & $3^{2}$ & $3 \cdot 6578391763$ & NONE & & & \\
\hline $2171 \mathrm{E}$ & 54 & $13^{2}$ & $=$ & 271 & NONE & & & \\
\hline $2173 H$ & 44 & $199^{2}$ & $=$ & $199 \cdot 3581$ & 2173D & 2 & {$\left[398^{2}\right]$} & - \\
\hline $2173 F$ & 43 & $19^{2}$ & $3^{2} \cdot 19^{2}$ & $3^{2} \cdot 19 \cdot 229341$ & 2173A & 1 & {$\left[38^{2}\right]$} & $19^{2}$ \\
\hline $2174 F$ & 31 & $5^{2}$ & $=$ & $5 \cdot 21555702093188316107$ & NONE & & & \\
\hline 2181E & 27 & $7^{2}$ & $=$ & $7 \cdot 7217996450474835$ & 2181A & 1 & {$\left[28^{2}\right]$} & - \\
\hline $2193 K$ & 17 & $3^{2}$ & $=$ & $3 \cdot 15096035814223$ & $129 \mathrm{~A}$ & 1 & {$\left[21^{2}\right]$} & - \\
\hline $2199 \mathrm{C}$ & 36 & $7^{2}$ & $=$ & $7^{2} \cdot 13033437060276603$ & NONE & & & \\
\hline $2213 \mathrm{C}$ & 101 & $3^{4}$ & $=$ & 19 & NONE & & & \\
\hline $2215 \mathrm{~F}$ & 46 & $13^{2}$ & $=$ & $13 \cdot 1182141633$ & 2215A & 1 & {$\left[52^{2}\right]$} & - \\
\hline 2224R & 11 & $79^{2}$ & $=$ & 79 & $2224 G$ & 2 & {$\left[79^{2}\right]$} & - \\
\hline $2227 \mathrm{E}$ & 51 & $11^{2}$ & $=$ & 259 & NONE & & & \\
\hline 2231D & 60 & $47^{2}$ & $=$ & 91109 & NONE & & & \\
\hline 2239B & 110 & $11^{4}$ & $=$ & . & NONE & & & \\
\hline $2251 \mathrm{E} *$ & 99 & $37^{2}$ & $=$ & 37 & 2251A & 1 & {$\left[74^{2}\right]$} & $37^{2}$ \\
\hline $2253 \mathrm{C} *$ & 27 & $13^{2}$ & $=$ & $13 \cdot 14987929400988647$ & 2253A & 1 & {$\left[26^{2}\right]$} & $13^{2}$ \\
\hline $2255 J$ & 23 & $7^{2}$ & $=$ & 15666366543129 & NONE & & & \\
\hline $2257 \mathrm{H}$ & 46 & $3^{6} \cdot 29^{2}$ & $=$ & $3^{3} \cdot 29 \cdot 175$ & 2257A & 1 & {$\left[9^{2}\right]$} & - \\
\hline & & & & & 2257D & 2 & {$\left[2^{2} 174^{2}\right]$} & - \\
\hline 2264J & 22 & $73^{2}$ & $=$ & 73 & $2264 B$ & 2 & {$\left[146^{2}\right]$} & - \\
\hline $2265 U$ & 14 & $7^{2}$ & $=$ & $7^{2} \cdot 73023816368925$ & 2265B & 1 & {$\left[7^{2}\right]$} & - \\
\hline 2271I $*$ & 43 & $23^{2}$ & $=$ & $23 \cdot 392918345997771783$ & $2271 C$ & 1 & {$\left[46^{2}\right]$} & $23^{2}$ \\
\hline $2273 \mathrm{C}$ & 105 & $7^{2}$ & $=$ & $7^{2}$ & NONE & & & \\
\hline $2279 D$ & 61 & $13^{2}$ & $=$ & 96991 & NONE & & & \\
\hline $2279 \mathrm{C}$ & 58 & $5^{2}$ & $=$ & 1777847 & NONE & & & \\
\hline 2285E & 45 & $151^{2}$ & $=$ & $151 \cdot 138908751161$ & 2285A & 2 & {$\left[2^{2} 302^{2}\right]$} & - \\
\hline $2287 B$ & 109 & $71^{2}$ & $=$ & 1 & NONE & & & \\
\hline $2291 \mathrm{C}$ & 52 & $3^{2}$ & $=$ & 427943 & NONE & & & \\
\hline $2293 \mathrm{C}$ & 96 & $479^{2}$ & $=$ & 479 & 2293A & 2 & {$\left[2^{2} 958^{2}\right]$} & - \\
\hline $2294 \mathrm{~F}$ & 15 & $3^{2}$ & $=$ & $3 \cdot 6289390462793$ & $1147 \mathrm{~A}$ & 1 & {$\left[3^{2}\right]$} & - \\
\hline 2311B & 110 & $5^{2}$ & $=$ & 1 & NONE & & & \\
\hline $2315 I$ & 51 & $3^{2}$ & $=$ & $3 \cdot 4475437589723$ & $463 \mathrm{~A}$ & 16 & {$\left[13426312769169^{2}\right]$} & - \\
\hline $2333 \mathrm{C}$ & 101 & $83341^{2}$ & $=$ & 83341 & 2333A & 4 & {$\left[2^{6} 166682^{2}\right]$} & - \\
\hline
\end{tabular}


Define integers $S_{l}$ and $S_{u}$ such that

$$
S_{l}\left|\operatorname{numer}\left(\# \amalg_{?}^{\text {odd }}\right)\right| S_{u}
$$

as follows:

$\mathbf{S}_{u}$ : The upper bound $S_{u}$ is the odd part of the numerator of

$$
\frac{L(A, 1)}{\tilde{\Omega}_{A}} \cdot \frac{T^{2}}{\prod_{p \| N} c_{p}},
$$

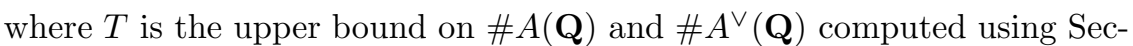
tion 3.5 and using $a_{p}$ for $p \leq 17$. Since the Manin constant and the Tamagawa numbers are integers, $S_{u}$ is an upper bound on the odd part of \# $\amalg_{\text {? }}$.

$\mathbf{S}_{l}$ : The lower bound $S_{l}$ is defined as follows: Let $S_{l, 1}$ be the odd part of the rational number

$$
\frac{L(A, 1)}{\tilde{\Omega}_{A}} \cdot \frac{\# C \cdot \# D}{\prod_{p \| N} c_{p}},
$$

where $C \subset A(\mathbf{Q})_{\text {tor }}$ and $D$ is the part of $C$ coprime to the modular degree of $A$. Usually $C$ is the group generated by the image of $(0)-(\infty)$, and in all cases it contains this subgroup. More precisely, when $A$ is an elliptic curve, we instead let $C$ and $D$ be the full torsion subgroup $A(\mathbf{Q})_{\text {tor }}$, because it is easy to calculate. When $A$ is not an elliptic curve, it would be better to let $C$ be the subgroup generated by all rational cusps, but the authors only realized this after completing the calculations, so we did not do this.

If $N$ is squarefree, we let $S_{l}=S_{l, 1}$. Otherwise, let $S_{l, 2}$ be the largest part of $S_{l, 1}$ coprime to all primes whose square divides $N$. This takes care of the Manin constant, which only involves primes whose square divides $N$. To take care of Tamagawa numbers, remove all primes $p \leq 2 \operatorname{dim}(A)+1$ from $S_{l, 2}$ to obtain $S_{l}$.

Remark 5.1. When $N$ is squarefree, we have

$$
S_{l}\left|\# \amalg_{?}^{\text {odd }}\right| S_{u}
$$

since $c_{A}$ is a power of 2 and no Tamagawa numbers have been omitted from the formulas for $S_{l}$ and $S_{u}$. For every $N \leq 2333$ we found that $S_{l}$ is an integer, so when $N \leq 2333$ is squarefree, \# $\amalg_{?}^{\text {odd }}$ is an integer. Since Conjecture 2.2 asserts that \# $\amalg_{\text {? }}$ is the order of a group, hence an integer, our data gives evidence for Conjecture 2.2.

Tables 14 list every $A$ of level $N \leq 2333$ such that $S_{l}>1$. The $A$ column contains the label of $A$ (see Section 3.2), and the next column (labeled dim) contains $\operatorname{dim} A$. A star next to the label for $A$ indicates that we have proved that the odd part of \# $\amalg(A)$ is at least as large as conjectured by the Birch and SwinnertonDyer conjecture. This is the case for 37 of the 168 examples. The column labeled $S_{l}$ contains the number $S_{l}$ defined above. If $S_{l}=S_{u}$, then the column labeled $S_{u}$ contains an $=$ sign, and otherwise it contains $S_{u}$ (there are only 13 cases in which $\left.S_{u} \neq S_{l}\right)$. The column labeled $\operatorname{moddeg}(A)^{\text {odd }}$ contains the odd part $m$ of the modular degree of $A$, written as a product $\operatorname{gcd}\left(m, S_{u}\right) \cdot m / \operatorname{gcd}\left(S_{u}, m\right)$, where $\mathrm{m} / \operatorname{gcd}\left(S_{u}, m\right)$ is shrunk to save space. The only nonsquarefree levels of $A_{f}$ for which $S_{l}>1$ are 1058, 1664, 2224, and 2264 . 
The column labeled $B$ contains all $B$ such that $L(B, 1)=0$ and

$$
\operatorname{gcd}\left(S_{l}, \#\left(A^{\vee} \cap \tilde{B}^{\vee}\right)\right)>1 .
$$

(In retrospect, it would probably have been more interesting to list those $B$ such that $\operatorname{gcd}\left(S_{u}, \#\left(A^{\vee} \cap \tilde{B}^{\vee}\right)\right)>1$.) Here $B=A_{g}$ for some newform $g$ of level dividing $N$, and $\tilde{B}^{\vee}$ is the abelian subvariety of $J_{0}(N)$ generated by all images of $B^{\vee}$ under the degeneracy maps. Thus, e.g., when $B^{\vee}$ is of level $N, \tilde{B}^{\vee}=B^{\vee}$. The next column, labeled dim, contains the dimension of $B$.

The final two columns contain information about the relationship between $A$ and $B$. The one labeled $A^{\vee} \cap \tilde{B}^{\vee}$ contains the abelian group structure of the indicated abelian group, where, e.g., $\left[a^{b} c^{d}\right]$ means the abelian group $(\mathbf{Z} / a \mathbf{Z})^{b} \times$ $(\mathbf{Z} / c \mathbf{Z})^{d}$. The column labeled Vis contains a divisor of the order of $\operatorname{Vis}_{C}\left(\amalg\left(A^{\vee}\right)\right)$, where $C=A^{\vee}+\tilde{B}^{\vee}$ (note that $\operatorname{Vis}_{C}\left(\amalg\left(A^{\vee}\right)\right) \subset \operatorname{Vis}_{J_{0}(N)}\left(\amalg\left(A^{\vee}\right)\right)$ ).

The table is divided into three vertical regions, where the columns in the first region are about $A$ only, the columns of the second region are about $B$ only, and the third column is about the relationship between $A$ and $B$.

5.1. Example: Level 389. We illustrate what is involved in computing the first line of Table 1. Using the method sketched in Section 3.2, we find that $S_{2}\left(\Gamma_{0}(389)\right)$ contains exactly five Galois-conjugacy classes of newforms, and these are defined over extensions of $\mathbf{Q}$ of degrees $1,2,3,6$, and 20 . Thus $J=J_{0}(389)$ decomposes, up to isogeny, as a product $A_{1} \times A_{2} \times A_{3} \times A_{6} \times A_{20}$ of abelian varieties, where $\operatorname{dim} A_{d}=d$ and $A_{d}$ is the optimal quotient corresponding to the appropriate Galoisconjugacy class of newforms.

Next we consider the arithmetic of the $A_{d}$. Using Theorem 4.5, we find that

$$
L\left(A_{1}, 1\right)=L\left(A_{2}, 1\right)=L\left(A_{3}, 1\right)=L\left(A_{6}, 1\right)=0
$$

and

$$
\frac{L\left(A_{20}, 1\right)}{\Omega_{A_{20}}}=\frac{5^{2} \cdot 2^{11}}{97 \cdot c_{A}}
$$

where $c_{A}$ is the Manin constant attached to $A_{20}$, which, by Theorem 4.2 is of the form $2^{n}$ with $n \geq 0$. Using the algorithms of Sections 3.5, 3.6 3.7, we find that $\# A_{20}(\mathbf{Q})=c_{389}=97$. Thus Conjecture 2.2 predicts that \# $\amalg\left(A_{20}\right)=5^{2} \cdot 2^{11} / c_{A}$. The following proposition provides support for this conjecture.

Proposition 5.2. There is a natural inclusion

$$
(\mathbf{Z} / 5 \mathbf{Z})^{2} \cong A_{1}(\mathbf{Q}) / 5 A_{1}(\mathbf{Q}) \hookrightarrow \operatorname{Vis}_{J_{0}(389)}\left(\amalg\left(A_{20}^{\vee}\right)\right) .
$$

Proof. Let $A=A_{20}^{\vee}, B=A_{1}^{\vee}$ and $J=A+B \subset J_{0}(389)$. Using Proposition 3.3 we find that $A \cap B \cong(\mathbf{Z} / 4)^{2} \times(\mathbf{Z} / 5 \mathbf{Z})^{2}$, so $B[5] \subset A$. Since 5 does not divide the numerator of $(389-1) / 12$, it does not divide the Tamagawa numbers or the orders of the torsion groups, so Theorem 3.13 yields the asserted injection. To see that $(\mathbf{Z} / 5 \mathbf{Z})^{2} \cong A_{1}(\mathbf{Q}) / 5 A_{1}(\mathbf{Q})$, use the standard elliptic curves algorithms [Cre97].

5.2. Invisible elements of $\amalg(A)$. Tables 10 suggest that much of $\amalg\left(A^{\vee}\right)$ is invisible in $J_{0}(N)$. This is because Proposition 3.15 implies that if a prime divides \# $\amalg\left(A^{\vee}\right)$ but not moddeg $\left(A^{\vee}\right)$, then $\amalg\left(A^{\vee}\right)$ contains an element of order $p$ that is invisible. We find many examples in the table where $p$ divides the conjectural order of $\amalg\left(A^{\vee}\right)$, but $p \nmid \operatorname{moddeg}\left(A^{\vee}\right)$.

Invisible elements might become visible at higher level (see [AS02, §4.3] for a discussion and example). 
5.3. The part of $\amalg(A)$ that must be a perfect square. When $\operatorname{dim} A=1$, properties of the Cassels-Tate pairing imply that if $\amalg(A)$ is finite, then \# $\amalg(A)$ is a perfect square, and the fact that one finds in examples (see [Cre97]) that \# Ш is a perfect square is computational evidence for Conjecture 2.2.

In contrast, when the dimension is greater than one, Poonen and Stoll PS99] discovered Jacobians $J$ such that $\amalg(J)$ has order twice a square, and the second author found for each prime $p<25000$ an abelian variety $A$ of dimension $p-1$ such that \# $\amalg(A)=p n^{2}$ for some integer $n$ (see Ste02b]).

Proposition 5.3. Let $A=A_{f}$ be a quotient of $J_{0}(N)$ and let $\ell$ be a prime that does not divide the modular degree of $A$. Suppose that $\amalg(A)\left[\ell^{\infty}\right]$ is finite. Then \# $\amalg(A)\left[\ell^{\infty}\right]$ is a perfect square.

Proof. The Cassels-Tate pairing (see Tat63 §3]) induces a pairing

$$
\phi: \amalg(A)\left[\ell^{\infty}\right] \times \amalg\left(A^{\vee}\right)\left[\ell^{\infty}\right] \rightarrow \mathbf{Q} / \mathbf{Z} .
$$

Since $\amalg(A)\left[\ell^{\infty}\right]$ is finite, it follows from Tat63. Thm. 3.2] that $\amalg\left(A^{\vee}\right)\left[\ell^{\infty}\right]$ is also finite and $\phi$ is nondegenerate. In particular, \# $\amalg\left(A^{\vee}\right)\left[\ell^{\infty}\right]=\# \amalg(A)\left[\ell^{\infty}\right]$.

Since $J_{0}(N)$ is a Jacobian, it possesses a canonical polarization arising from the theta divisor; this divisor is rational over $\mathbf{Q}$, since $X_{0}(N)$ always has a point over $\mathbf{Q}$ (the cusp $\infty$ is rational). This polarization induces a polarization $\theta: A^{\vee} \rightarrow A$, which also comes from a divisor that is rational over $\mathbf{Q}$. Hence, by Tat63, Thm. 3.3] (see also [PS99, Thm. 5]), the pairing

$$
\phi^{\prime}: \amalg\left(A^{\vee}\right)\left[\ell^{\infty}\right] \times \amalg\left(A^{\vee}\right)\left[\ell^{\infty}\right] \rightarrow \mathbf{Q} / \mathbf{Z}
$$

obtained by composing $\theta$ with the pairing $\phi$ above is alternating.

Since $\ell$ does not divide the modular degree of $A$, it does not divide the degree of the isogeny $\theta$. Hence $\theta$ induces an isomorphism $\amalg\left(A^{\vee}\right)\left[\ell^{\infty}\right] \cong \amalg(A)\left[\ell^{\infty}\right]$. Thus by the nondegeneracy of the pairing $\phi$, the pairing $\phi^{\prime}$ is also nondegenerate. Since $\phi^{\prime}$ is also alternating, it follows from arguments similar to those in [Cas62, p. 260] that \# $\amalg\left(A^{\vee}\right)\left[\ell^{\infty}\right]$ is a perfect square. Since \# $\amalg(A)\left[\ell^{\infty}\right]=\# \amalg\left(A^{\vee}\right)\left[\ell^{\infty}\right]$, we see that \# $\amalg(A)\left[\ell^{\infty}\right]$ is also a perfect square.

For the entries in Tables 14 $\amalg(A)$ is finite, so if $\ell \nmid \operatorname{moddeg}(A)$, then the $\ell$-power part of \# $\amalg(A)$ must be a perfect square. When $S_{l}=S_{u}$ and the level is squarefree, then $S_{l}$ is the odd part of the conjectural order of $\amalg(A)$. We found that $S_{l}$ is a perfect square whenever $S_{l}=S_{u}$, which provides evidence for Conjecture 2.2 


\section{APPENDIX. \\ "Explaining" Shafarevich-Tate via Mordell-Weil}

In our article [CM] we discussed the notion of visibility and offered some tables of examples of that phenomenon. We gave, however, very little theoretical discussion in that article. Here we wish to take the opportunity to correct some gaps in our commentary on our tables and to offer the details of the proof of a general criterion that is sometimes useful to test visibility. Regarding Table 1 of $[\mathrm{CM}]$ we said that for each pair $(E, p)$ that occurs there and for which there is a corresponding " $F$ " on the table of the same conductor of $E$, the Shafarevich-Tate group of $E$ is explained by the Mordell-Weil group of $F$, in the technical sense that we gave to the word explained in that article. Now this is indeed the case for all entries of our table such that $E$ has semistable reduction at $p$ and it is also the case for those entries where the conductor of $F$ properly divides the conductor of $E$. We will review why this is so, below. It is also true that for each of the remaining 7 entries $(E=$ 2601H, 2718D, 2900D, 3555E, 3879E, 3933A, 5499E) a nontrivial subgroup of the Shafarevich-Tate group of $E$ is explained by the Mordell-Weil group of the corresponding $F$, but we wish to notify our readers that we have not yet checked whether or not all of the " $\amalg$ " of these 7 elliptic curves is so explained. These 7 cases deserve to be looked at (the issue being local at the prime 3 for all but 2900D, where it is local at the prime 5). Regarding Table 2 of [CM], although our commentary in $[\mathrm{CM}]$ does not say this clearly, for all the entries $E$ of that table for which there is a corresponding $F$ of the same conductor we only have checked that $E[2]=F[2]$ in $J_{0}(N)$ and nothing more, except, of course, for those entries we particularly signal to have shown something less, namely, in the language of our article, that they "seem to satisfy a 2-congruence." In these latter cases where we signal that we have shown something less, W. Stein has checked that in fact $E[2] \neq F[2]$ in $J_{0}(N)$.

Let $p$ be an odd prime number. If $E$ is an (optimal) elliptic curve over $\mathbf{Q}$ of conductor $N$, then $E$ may be unambiguously identified (up to sign) with a subabelian variety of the modular Jacobian $J_{0}(N)$ (over $\mathbf{Q}$ ). If $(E, F, p)$ is an entry of Table 1 of $[\mathrm{CM}]$ such that $E$ and $F$ are of the same conductor $N$, we checked that we have equality of the finite group schemes $E[p]_{/ \mathbf{Q}}=F[p]_{/ \mathbf{Q}}$ in $J_{0}(N)_{/ \mathbf{Q}}$. For the remaining three entries, we checked that there is an isomorphism of finite group schemes $\iota: E[p]_{/ \mathbf{Q}} \cong F[p]_{/ \mathbf{Q}}$. In both cases, identifying the two finite group schemes, let $H$ denote the common cohomology group,

$$
H:=H^{1}\left(G_{\mathbf{Q}}, E[p]_{/ \mathbf{Q}}\right)=H^{1}\left(G_{\mathbf{Q}}, F[p]_{/ \mathbf{Q}}\right),
$$

and let $S_{E} \subset H$ and $S_{F} \subset H$ be the $p$-Selmer groups of, respectively, $E$ and $F$. What we will show is that

Proposition A.1. For each of the entries (E,p) in Table 1 of [CM] such that $p$ is a prime of semistable reduction for $E$ and for which there is a "corresponding" $F$, we have

$$
S_{E}=S_{F} \subset H .
$$

To discuss this, we need some notation.

Let $X:=\operatorname{Spec}(\mathbf{Z}), Y:=\operatorname{Spec}(\mathbf{Z}[1 / p])=X-\operatorname{Spec}\left(\mathbf{F}_{p}\right)$, and $\eta:=\operatorname{Spec}(\mathbf{Q})$. Let $E_{\eta}:=E$ be our elliptic curve over $\mathbf{Q}$ of conductor $N, E_{/ X}$ the Néron model over $X$ of $E_{\eta}$ and $E_{/ X}^{o} \subset E_{/ X}$ the "connected component" of Néron (meaning the 
open subgroup scheme every fiber of which is connected). We have, of course, similar notation for the corresponding elliptic curve $F$. Let $E[p]_{/ X}$ denote the closed subgroup scheme given as the kernel of multiplication by $p$ in the Néron model: $E[p]_{/ X} \subset E_{/ X}$. We have, in general, that the restriction $E[p]_{/ Y}$ of $E[p]_{/ X}$ to the base $Y$ is an étale quasi-finite flat group scheme; and if $p^{2}$ does not divide $N$, we have that the group scheme $E[p] / X$ is a quasi-finite flat group scheme Gro72. Prop. 3.1(d), p. 343]. The étale quasi-finite flat group scheme $E[p]_{/ Y}$ can be characterized by the following features:

(i) Its generic fiber is the group scheme $E[p]_{/ \eta} \subset J_{0}(N) / \eta$, and (one has a choice here) either

(ii) $E[p]_{/ Y} \subset J_{0}(N)_{/ Y}$ is a closed étale quasi-finite flat subgroup scheme

or

(ii') $E[p]_{/ Y}$ enjoys the Néronian property over the base $Y$.

Similar statements hold for $F[p] / X$.

Let $\Phi$ be the (punctual) sheaf of abelian groups for the flat topology over $X$ which fits into the exact sequence (of abelian sheaves over $X$ )

$$
0 \rightarrow E^{o} \rightarrow E \rightarrow \Phi \rightarrow 0 .
$$

We will use the same notation to indicate the corresponding exact sequence of sheaves for the étale topology over $X$. Since $E^{0}$ and $E$ are smooth group schemes, the long exact sequences of cohomology derived from the short exact sequence (A.1), viewed either as sheaves of abelian groups for the flat or étale topology, coincide; cf Section 11, Appendice: Un théorème de comparaison de la cohomologie étale et de la cohomologie fppf in Gro68]. Thinking now of $\Phi$ as a sheaf for the étale topology, denote by $\Phi_{\ell}$ its stalk at the prime $\ell$. So $\Phi_{\ell}$ is representable as a finite étale group scheme over the field $\mathbf{F}_{\ell}$. We have that

$$
\Phi=\bigoplus_{\ell \mid N}\left(i_{\ell}\right)_{*} \Phi_{\ell},
$$

where $i_{\ell}: \operatorname{Spec} \mathbf{F}_{\ell} \hookrightarrow X$ is the natural closed immersion. We have an exact sequence $(\mathrm{A} .2) 0 \rightarrow E^{o}(X) \rightarrow E(\mathbf{Q}) \rightarrow H^{0}(X, \Phi) \rightarrow H^{1}\left(X, E^{o}\right) \rightarrow H^{1}(X, E) \rightarrow H^{1}(X, \Phi)$, where cohomology is computed for the étale topology. We have, for either topology,

$$
H^{i}(X, \Phi)=\bigoplus_{\ell \mid N} H^{i}\left(\operatorname{Spec}\left(\mathbf{F}_{\ell}\right), \Phi_{\ell}\right)
$$

Viewing A.1 as an exact sequence of sheaves for the flat topology and passing to the associated cohomology sequence, we see that A.2 may be thought of ambiguously as computed for either the étale or the flat topology.

If $p$ is an odd prime number, the $p$-primary component of the Shafarevich-Tate group of $E$ is the $p$-primary component of the image of $H^{1}\left(X, E^{o}\right) \rightarrow H^{1}(X, E)$ (see the appendix to [Maz72]) or equivalently the intersection of the kernels of

$$
H^{1}(X, E) \rightarrow H^{1}\left(\operatorname{Spec}\left(\mathbf{F}_{\ell}\right), \Phi_{\ell}\right) .
$$

Let $p$ be an odd prime number. Let $E^{\prime} \subset E$ be the open subgroup scheme of $E$ which is the inverse image of $p \Phi \subset \Phi$, so that we have an exact sequence of sheaves for the flat (or étale) topology,

$$
0 \rightarrow E^{\prime} \rightarrow E \rightarrow \Phi / p \Phi \rightarrow 0
$$


and if $p$ is a prime of semistable reduction for $E$ (equivalently, $p^{2}$ does not divide $N$ ), we have an exact sequence of flat group schemes

$$
0 \rightarrow E[p] \rightarrow E \rightarrow E^{\prime} \rightarrow 0 .
$$

Put

$$
E[p]_{/ X}^{o}:=E[p]_{/ X} \bigcap E_{/ X}^{o}
$$

Then $E[p]_{/ X}^{o}$ is an open (quasi-finite) subgroup scheme of $E[p]_{/ X}$. Let $\tilde{E}[p]_{/ X}$ be any "intermediate" open (quasi-finite) subgroup scheme

$$
E[p]_{/ X}^{o} \subset \tilde{E}[p]_{/ X} \subset E[p]_{/ X}
$$

so that we have the exact sequence of sheaves for the finite flat topology

$$
0 \rightarrow \tilde{E}[p]_{/ X} \rightarrow E[p]_{/ X} \rightarrow \Psi \rightarrow 0
$$

with $\Psi$ a subquotient of $\Phi$.

Consider the following hypothesis:

$\mathbf{A}(E, p, \ell)$ : The Galois module $\Phi_{\ell} / p \Phi_{\ell}$ is either trivial or else it is a nonconstant cyclic Galois module over $\mathbf{F}_{\ell}$.

Let $\mathbf{A}(E, p)$ denote the conjunction of hypothesis $\mathbf{A}(E, p, \ell)$ for all prime numbers $\ell$, or equivalently, for all $\ell$ dividing $N$.

Lemma A.2. These are equivalent formulations of hypothesis $\mathbf{A}(E, p)$.

(a) $\Phi / p \Phi$ is cohomologically trivial; that is, $H^{0}(X, \Phi / p \Phi)=H^{1}(X, \Phi / p \Phi)=0$.

(b) If $\Psi$ is any subquotient of $\Phi, \Psi$ is "p-cohomologically trivial" in the sense that the p-primary components of $H^{i}(X, \Psi)$ vanish for all $i$.

Moreover, if $p \geq 5$, or if $p=3$ and $E$ has no Néron fibers of type $I V$ or $I V^{*}$, the above conditions are equivalent to

(c) For every $\ell$ at which $E$ has split multiplicative reduction, $p$ does not divide the order of the group of connected components of the Néron fiber of $E$ at $\ell$.

Proof. The equivalence of hypothesis $\mathbf{A}(E, p)$ with (a) and with (b) is straightforward using standard exact sequences plus the fact that the $p$-primary components of the (underlying abelian group of) $\Phi_{\ell}$ is cyclic since $p>2$, and noting that a (finite) $G$-module of prime order with nontrivial $G$-action has trivial cohomology. For (c) we are using that if $p>2$, the $p$-primary component of $\Phi_{\ell}$ vanishes for all primes $\ell$ of additive reduction for $E$ except when $p=3$ and the Néron fiber type of $E$ at $\ell$ is IV or IV*.

A morphism $G_{1} \rightarrow G_{2}$ of flat (commutative, finite type) group schemes over $X$ will be said to induce an isomorphism on p-cohomology if the induced mappings

$$
H^{i}\left(X, G_{1}\right) \otimes \mathbf{Z}_{p} \rightarrow H^{i}\left(X, G_{2}\right) \otimes \mathbf{Z}_{p}
$$

are isomorphisms for all $i \geq 0$, where cohomology is computed for the flat topology.

Lemma A.3. Let $p$ be an odd prime number for which $\mathbf{A}(E, p)$ holds. We have that the natural morphisms

$$
E_{/ X}^{o} \rightarrow E_{/ X}^{\prime} \quad \text { and } \quad E_{/ X}^{\prime} \rightarrow E_{/ X}
$$

induce isomorphisms on p-cohomology. If $p$ is of semistable reduction for $E$, we also have that

$$
\tilde{E}[p]_{/ X} \rightarrow E[p]_{/ X}
$$


induces isomorphisms on p-cohomology, for any of the open subgroup schemes $\tilde{E}[p]_{/ X}$ in $E[p]_{/ X}$ described above.

Proof. These all sit in short exact sequences of sheaves of abelian groups for the flat topology over $X$ where the third sheaf is $p$-cohomologically trivial.

Corollary A.4. If $p>2$ and $\mathbf{A}(E, p)$ holds, we have natural isomorphisms

$$
H^{0}\left(X, E^{o}\right) \otimes \mathbf{Z}_{p} \cong H^{0}\left(X, E^{\prime}\right) \otimes \mathbf{Z}_{p} \cong E(\mathbf{Q}) \otimes \mathbf{Z}_{p}
$$

and

$$
\amalg(E) \otimes \mathbf{Z}_{p} \cong H^{1}\left(X, E^{o}\right) \otimes \mathbf{Z}_{p} \cong H^{1}\left(X, E^{\prime}\right) \otimes \mathbf{Z}_{p} \cong H^{1}(X, E) \otimes \mathbf{Z}_{p} .
$$

Corollary A.5. Let $p$ be an odd prime number, semistable for $E$, and suppose that $\mathbf{A}(E, p)$ holds.

(i) The image of the natural (injective) coboundary mapping

$$
0 \rightarrow E(\mathbf{Q}) / p E(\mathbf{Q}) \hookrightarrow H^{1}\left(G_{\mathbf{Q}}, E[p]\right)
$$

attached to the Kummer sequence is contained in the image of the natural injection

$$
H^{1}\left(X, E[p]^{o}\right) \hookrightarrow H^{1}\left(G_{\mathbf{Q}}, E[p]\right) .
$$

(ii) We have an exact sequence

$$
0 \rightarrow E(\mathbf{Q}) / p E(\mathbf{Q}) \rightarrow H^{1}(X, \tilde{E}[p]) \rightarrow \amalg(E)[p] \rightarrow 0
$$

for any of the open subgroup schemes $\tilde{E}[p]_{/ X} \subset E[p]_{/ X}$ defined above.

(iii) The image of $H^{1}(X, \tilde{E}[p]) \hookrightarrow H^{1}\left(G_{\mathbf{Q}}, E[p]\right)$ is equal to the $p$-Selmer subgroup,

$$
S_{p}(E) \subset H^{1}\left(G_{\mathbf{Q}}, E[p]\right) .
$$

Proof. All this follows from straightforward calculations using the cohomological exact sequences associated to the exact sequences (1)-(5) in the light of the previous discussion.

To set things up for our application, let us record the following:

Corollary A.6. Let $E_{/ \mathbf{Q}}$ and $F_{/ \mathbf{Q}}$ be elliptic curves over $\mathbf{Q}$. Let $p$ be an odd prime number of semistable reduction for $E$ and $F$ and for which $\mathbf{A}(E, p)$ and $\mathbf{A}(F, p)$ both hold. Define $\tilde{E}[p]_{/ X} \subset E[p]_{/ X}$ to be the open quasi-finite subgroup scheme whose restriction to $Y$ is equal to $E[p]_{/ Y}$ and whose fiber at $\mathbf{F}_{p}$ is equal to $E[p]_{/ \mathbf{F}_{p}}^{o}=\left(E[p] \cap E^{o}\right)_{/ \mathbf{F}_{p}}$. Define $\tilde{F}[p]_{/ X}$ similarly. Suppose, finally, that we have an isomorphism of $G_{\mathbf{Q}}$-modules $\iota: F[p]_{/ \mathbf{Q}} \cong E[p]_{/ \mathbf{Q}}$ which extends to an injection of quasi-finite flat group schemes

$$
\tilde{F}[p]_{/ X} \hookrightarrow \tilde{E}[p]_{/ X}
$$

Letting

$$
H:=H^{1}\left(G_{\mathbf{Q}}, E[p]\right)=H^{1}\left(G_{\mathbf{Q}}, F[p]\right)
$$

(making the identification via $\iota)$, we have that the $p$-Selmer groups $S_{p}(E) \subset H$ and $S_{p}(F) \subset H$ are the same. 
Proposition A.7. Let $(E, F, p)$ be a triple which is an entry of Table 1 of [CM]. Suppose further that $p$ is of semistable reduction for $E$ and for $F$. Then, with the notation of the previous corollary, the p-Selmer groups $S_{p}(E) \subset H$ and $S_{p}(F) \subset$ $H$ are the same. In the terminology of [CM] the Shafarevich-Tate group of $E$ is explained by the Mordell-Weil group of F.

Proof. As mentioned above, we have checked that $E[p]_{/ \mathbf{Q}}=F[p]_{/ \mathbf{Q}} \subset J_{0}(N)$ whenever the pair $E$ and $F$ (appearing as an entry of Table 1 of [CM]) have the same conductor. We have checked that $E[p]_{/ \mathbf{Q}} \cong F[p]_{/ \mathbf{Q}}$ for the three entries where $E$ and $F$ have different conductor $(E=\mathbf{2 9 3 2 A}, \mathbf{3 3 0 6 B}$, and 5136B $)$. We have checked that hypotheses $A(E, p, \ell)$ and $A(F, p, \ell)$ hold for all quadruples $(E, F, p, \ell)$ such that $(E, F, p)$ occurs as an entry in Table 1 of $[\mathrm{CM}$ ] (even when $p$ is not semistable for $E$ and $F)$ with the exception of the entry $(E, F, p, \ell)=(\mathbf{2 3 6 6 D}, \mathbf{2 3 6 6 \mathbf { E }}, 3,13)$.

Sublemma A.8. Under the hypotheses of our proposition, the isomorphism of $G_{\mathbf{Q}}$-modules $\iota: E[p]_{/ \mathbf{Q}} \cong F[p]_{/ \mathbf{Q}}$ extends to an injection of quasi-finite flat group schemes

$$
\tilde{E}[p]_{/ X} \hookrightarrow \tilde{F}[p]_{/ X}
$$

which is an isomorphism except in two instances $(E=3306 \mathrm{~B}$ and $5136 \mathrm{~B})$.

Proof. First, since $\tilde{E}[p]_{/ Y}=E[p]_{/ Y}, \tilde{F}[p]_{/ Y}=F[p]_{/ Y}$, and, as we mentioned at the beginning, both of these quasi-finite, flat (étale) group schemes $F[p]_{/ Y}$ and $E[p]_{/ Y}$ enjoy the Néronian property, the isomorphism $\iota$ extends to an isomorphism $\tilde{E}[p]_{/ Y} \cong \tilde{F}[p]_{/ Y}$. The remaining question is then local about $p$. If $p$ is of good reduction for $E$, then $\tilde{E}[p]_{/ X_{p}}$ and $\tilde{F}[p]_{/ X_{p}}$ are both finite flat group schemes of odd order, so by Fontaine's Theorem [Fon75], the isomorphism between their generic fibers extends to an isomorphism over $X_{p}$. (Compare Theorem I.1.4 in Maz77.) A standard result allows us to patch the isomorphism extending $\iota$ over $Y$ with the isomorphism ("extending $\iota$ ") over $X_{p}$ to get the extension of $\iota$ to an isomorphism of group schemes over $X, \tilde{E}[p]_{/ X} \cong \tilde{F}[p]_{/ X}$. Now consider the case where $p$ is of bad reduction. By the assumptions of our proposition, $p$ is then of multiplicative reduction for $E$, and hence the fiber of $E$ over $\mathbf{F}_{p}$ is a finite multiplicative type group scheme of order $p$. We therefore have that $\tilde{E}[p]_{/ X_{p}}$ sits in an exact sequence

$$
0 \rightarrow \mathcal{C}_{/ X_{p}} \rightarrow \tilde{E}[p]_{/ X_{p}} \rightarrow \mathcal{E}_{/ X_{p}} \rightarrow 0
$$

where $\mathcal{C}_{/ X_{p}}$ is a finite flat group scheme of order $p$ (and with fiber of multiplicative type in characteristic $p$ ) and where $\mathcal{E}_{/ X_{p}}$ is an étale quasi-finite group scheme, with trivial fiber in characteristic $p$.

Let us take a moment to recall (see [Maz78, Lem. 1.1]) the construction of such an exact sequence A.6): working in the category of formal schemes, let $\hat{X}_{p}:=\operatorname{Spf}\left(\mathbf{Z}_{p}\right)$, and let $\hat{\mathcal{C}}_{/ \hat{X}_{p}}$ be the formal completion of the zero-section in $\tilde{E}[p] / X_{p}$. One checks that $\hat{\mathcal{C}}_{/ \hat{X}_{p}}$ may be identified with a finite flat formal group scheme over $\hat{X}_{p}$ which admits a closed immersion into the formal group scheme over $\hat{X}_{p}$ associated to $\tilde{E}[p]_{/ X_{p}}$. A standard algebrization argument establishes that there is a (unique) finite flat subgroup scheme $\mathcal{C}_{/ X_{p}} \subset \tilde{E}[p]_{/ X_{p}}$ whose associated formal group scheme over $\hat{X}_{p}$ is $\hat{\mathcal{C}}_{/ \hat{X}_{p}}$. The exact sequence (A.6) is then obtained by letting $\mathcal{E}_{/ X_{p}}$ be the evident quotient (quasi-finite flat) group scheme and noting that, by construction, its special fiber is trivial. 
Now let us return to the proof of the sublemma. Since the restriction of $\iota$ to $\mathcal{C}_{/ X_{p}}$ (a finite flat multiplicative type group scheme of order $p$ ) is injective over the generic point, it follows (by elementary considerations, or by Fontaine's Theorem cited above) that $\iota$ restricted to $\mathcal{C}_{/ X_{p}}$ is an injection over $X_{p}$. Since $\mathcal{E}_{/ X_{p}}$ has trivial fiber in characteristic $p, \iota$ is an injection as was to be proved. In all cases under consideration, then,

$$
\iota: \tilde{E}[p]_{/ X} \hookrightarrow \tilde{F}[p] / X
$$

is an injection. If $E$ is of good reduction at $p$, or if $F$ is of bad reduction at $p, \iota$ is therefore an isomorphism. The cases remaining are when $E$ is of bad reduction at $p$ and $F$ is of good reduction (i.e., $E=3306 \mathrm{~B}$ and 5136B) in which case we can only assert that $\iota$ is an injection.

Returning to our proposition, suppose that $\tilde{F}[p]_{/ X_{p}}$ is finite flat (which happens in the two cases signalled above: $E=\mathbf{3 3 0 6 B}$ and $\mathbf{5 1 3 6 B}$ ). Then the isomorphism induced by $\iota$ on generic fibers

$$
\tilde{E}[p]_{/ \mathbf{Q}_{p}} \cong \tilde{F}[p]_{/ \mathbf{Q}_{p}}
$$

restricted to the $G_{\mathbf{Q}_{p}}$-stable subgroup $\mathcal{C}_{/ \mathbf{Q}_{p}} \subset \tilde{E}[p] / \mathbf{Q}_{p}$ extends to a morphism of the finite flat group scheme $\mathcal{C}_{/ X_{p}}$ into $\tilde{F}[p]_{/ X_{p}}$. This extended morphism $j: \mathcal{C}_{/ X_{p}} \rightarrow$ $\tilde{F}[p]_{X_{p}}$ is necessarily a closed immersion since $\mathcal{C}_{/ X_{p}}$ is a multiplicative type finite flat group scheme. Since $\mathcal{E}_{/ X_{p}}$ has trivial fiber in characteristic $p$, an application of the standard patching argument (as used in the previous case) allows us to put together the isomorphism of group schemes over $Y$ extending $\iota$ with the closed immersion $j$ over $X_{p}$ to get a closed immersion

$$
\tilde{E}[p]_{/ X} \hookrightarrow \tilde{F}[p]_{/ X}
$$

Finally suppose that both $E$ and $F$ have multiplicative reduction at $p$. We then have exact sequences (A.6) for each of our quasi-finite flat group schemes $\tilde{E}[p]_{/ X_{p}}$ and $\tilde{F}[p]_{/ X_{p}}$. Let $V$ denote their common generic fiber (identified via $\iota$ ) considered as a two-dimensional $\mathbf{F}_{p}$-vector space with $G_{\mathbf{Q}_{\mathrm{p}}}$-action. Let $\mathcal{C}(E) \subset V$ and $\mathcal{C}(F) \subset V$ denote the one-dimensional subspaces given by the generic fibers of the finite flat subgroup schemes $\mathcal{C}_{/ X_{p}}$ corresponding to the exact sequence (A.6) for $E$ and for $F$, respectively. Suppose, first, that these one-dimensional $\mathbf{F}_{p}$-subspaces $\mathcal{C}(E)$ and $\mathcal{C}(F)$ are different. It then follows that the $G_{\mathbf{Q}_{p}}$-representation $V$ splits as the direct sum of $\mathcal{C}(E)$ and $\mathcal{C}(F)$, both $\mathbf{F}_{p}$-subspaces being isomorphic, as $I_{\mathrm{Q}_{\mathrm{p}}}$-modules to $\mu_{p}$, where $I_{\mathbf{Q}_{\mathbf{p}}} \subset G_{\mathbf{Q}_{\mathbf{p}}}$ is the inertia subgroup of $G_{\mathbf{Q}_{p}}$. But this contradicts the fact that $V$ is self-Cartier dual (under the Weil pairing). Consequently, $\mathcal{C}(E)=\mathcal{C}(F) \subset$ $V$. From the above discussion it follows that we can extend $\iota$ to an isomorphism $\tilde{E}[p]_{/ X_{p}} \cong \tilde{F}[p]_{/ X_{p}}$.

Our proposition then follows (from Corollary A.6) for all entries in Table 1 of CM] where $p$ is of semistable reduction for $E$ once we produce special arguments to cover the three special cases $E=3306 \mathrm{~B}, \mathbf{5 1 3 6 \mathrm { B }}$ and $2366 \mathrm{D}$. The first two of these cases are "special" because we only have an injection $\tilde{E}[p] / X_{p} \hookrightarrow \tilde{F}[p] / X_{p}$ and not an isomorphism. However, the cokernel of this morphism restricted to the fiber in characteristic 3 is, in both of these cases, a cyclic group with nontrivial $G_{\mathbf{Q}_{3}}$ action and hence is 3-cohomologically trivial. In particular, the injection $\tilde{E}[p] / X_{p} \hookrightarrow$ $\tilde{F}[p]_{/ X_{p}}$ induces an isomorphism on flat cohomology over $X$, and the argument for

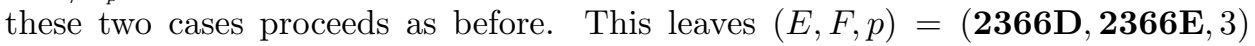


which is the only example of an entry $(E, F, p)$ in our table, where $E$ has a Qrational point of order $p$ and (this is no accident) where hypothesis $\mathbf{A}(E, p)$ and hypothesis $\mathbf{A}(F, p)$ fail. (Indeed there are no other failures of hypothesis $\mathbf{A}(F, p)$ for any of the $(E, F, p)$ 's occurring in Table 1 of $[\overline{\mathrm{CM}}]$ and only one other failure of hypothesis $\mathbf{A}(E, p)$, which is for $(E, p, \ell)=(\mathbf{2 9 3 2} \mathbf{A}, 3,2)$.)

Let us now deal with the case $(E, F, p)=(\mathbf{2 3 6 6 \mathbf { D }}, \mathbf{2 3 6 6 \mathbf { E }}, 3)$. The subgroup $C$ of Q-rational points of order 3 on $E$ specializes in characteristic 13 to yield an isomorphism

$$
C \cong \Phi_{13}
$$

and the same for the subgroup of Q-rational points of order 3 on $F$. We make use of this information to cut down the group schemes $\tilde{E}[3]_{/ X}$ and $\tilde{F}[3] / X$ and we define open subgroup schemes $\tilde{\tilde{E}}[3]_{/ X} \subset \tilde{E}[3]_{/ X}$ and $\tilde{\tilde{E}}[3]_{/ X} \subset \tilde{E}[3]_{/ X}$ by requiring that these closed immersions of subgroup schemes be isomorphisms outside characteristic 13 and that the "double-tilded" group schemes each have trivial fiber in characteristic 13. We get via the above argument an isomorphism of group schemes $\tilde{\tilde{E}}[3]_{/ X} \cong \tilde{\tilde{F}}[3]_{/ X}$ extending $\iota$ and an identification of the 3-Selmer groups of $E$ and $F$ with $H^{1}(X, \tilde{\tilde{E}}[3])$ and $H^{1}(X, \tilde{\tilde{F}}[3])$, respectively. Our proposition is proved.

It remains to say a few words about why, in the 7 cases of entries $(E, F, p)$ in our Table 1 of $[\mathrm{CM}]$ for which $p$ is a prime of additive reduction for $E$ some nontrivial elements of the Shafarevich-Tate group of $E$ are explained by the Mordell-Weil group of $F$. Briefly, the reason is as follows. By the inflated p-Selmer group of $E$ (and of $F$ ) let us mean the subgroup of $H$ obtained by insisting upon all the local Selmer conditions at primes different from $p$, but putting no condition at $p$. The $p$ Selmer group of $E$ (and of $F$ ) are, in all 7 instances, $\mathbf{F}_{p}$-vector spaces of dimension 2 and therefore, the inflated $p$-Selmer groups are of dimensions either 2 or 3 . Working over $Y$ rather than over $X$, the above argument applied to these 7 remaining cases gives us an identification of the inflated $p$-Selmer groups of $E$ and of $F$ in $H$. But the true $p$-Selmer groups (vector spaces of dimension 2) being subspaces in a vector space of dimension $\leq 3$ must have a nontrivial intersection.

\section{REFERENCES}

[Aga99] A. Agashe, On invisible elements of the Tate-Shafarevich group, C. R. Acad. Sci. Paris Sér. I Math. 328 (1999), no. 5, 369-374. MF2000e:11083

[AS] A. Agashe and W. A. Stein, Appendix to Joan-C. Lario and René Schoof: Some computations with Hecke rings and deformation rings, to appear in J. Exp. Math.

[AS02] A. Agashe and W. A. Stein, Visibility of Shafarevich-Tate Groups of Abelian Varieties, to appear in J. of Number Theory (2002).

[AS04] A. Agashe and W.A. Stein, The Manin constant, congruence primes, and the modular degree, preprint, (2004). http://modular.fas.harvard.edu/papers/manin-agashe/

[BCP97] W. Bosma, J. Cannon, and C. Playoust, The Magma algebra system. I. The user language, J. Symbolic Comput. 24 (1997), no. 3-4, 235-265, Computational algebra and number theory (London, 1993).

[Bir71] B. J. Birch, Elliptic curves over Q: A progress report, 1969 Number Theory Institute (Proc. Sympos. Pure Math., Vol. XX, State Univ. New York, Stony Brook, N.Y., 1969), Amer. Math. Soc., Providence, R.I., 1971, pp. 396-400. MR47:3395

[BLR90] S. Bosch, W. Lütkebohmert, and M. Raynaud, Néron models, Springer-Verlag, Berlin, 1990. MR 91i:14034

[Cas62] J. W.S. Cassels, "Arithmetic on curves of genus 1. III. The Tate-Šafarevič and Selmer groups", Proc. London Math. Soc. (3) 12 (1962), 259-296. MF 29:1212 
[Cre97] J. E. Cremona, Algorithms for modular elliptic curves, second ed., Cambridge University Press, Cambridge, 1997. MR.99e:11068

$[\mathrm{CM}]$ J. E. Cremona and B. Mazur, Visualizing elements in the Shafarevich-Tate group, Experiment. Math. 9 (2000), no. 1, 13-28.

[CS01] B. Conrad and W. A. Stein, Component groups of purely toric quotients, Math. Res. Lett. 8 (2001), no. 5-6, 745-766. MR2003f:11087

[Del01] C. Delaunay, Heuristics on Tate-Shafarevitch groups of elliptic curves defined over $\mathbf{Q}$, Experiment. Math. 10 (2001), no. 2, 191-196.

[DI95] F. Diamond and J. Im, Modular forms and modular curves, Seminar on Fermat's Last Theorem, Providence, RI, 1995, pp. 39-133. MR97g:11044

[Edi91] B. Edixhoven, On the Manin constants of modular elliptic curves, Arithmetic algebraic geometry (Texel, 1989), Birkhäuser Boston, Boston, MA, 1991, pp. 25-39. MR92a:11066

[Eme01] M. Emerton, Optimal quotients of modular Jacobians. Preprint.

[FpS:01] E. V. Flynn, F. Leprévost, E. F. Schaefer, W. A. Stein, M. Stoll, and J. L. Wetherell, Empirical evidence for the Birch and Swinnerton-Dyer conjectures for modular Jacobians of genus 2 curves, Math. Comp. 70 (2001), no. 236, 1675-1697.

[Fon75] J-M. Fontaine, Groupes finis commutatifs sur les vecteurs de Witt, C. R. Acad. Sci. Paris Sér. A-B 280 (1975), Ai, A1423-A1425. MR51:10353

[Gro94] B. H. Gross, L-functions at the central critical point, Motives (Seattle, WA, 1991), Amer. Math. Soc., Providence, RI, 1994, pp. 527-535. MR 95a:11060

[Gro68] A. Grothendieck, Le groupe de Brauer. III. Exemples et compléments, Dix Exposés sur la Cohomologie des Schémas, North-Holland, Amsterdam, 1968, pp. 88-188. MR39:5586c

[Gro72] A. Grothendieck, Modèles de Néron et monodromie in Groupes de monodromie en géométrie algébrique. I, Springer-Verlag, Berlin, 1972, Séminaire de Géométrie Algébrique du Bois-Marie 1967-1969 (SGA 7 I), Dirigé par A. Grothendieck. Vol. 288. MR.50:7134

[GZ86] B. Gross and D. Zagier, Heegner points and derivatives of L-series, Invent. Math. 84 (1986), no. 2, 225-320. MR87j:11057

[Kat81] N. M. Katz, Galois properties of torsion points on abelian varieties, Invent. Math. 62 (1981), no. 3, 481-502. MR82d:14025

[KL89] V.A. Kolyvagin and D. Y. Logachev, Finiteness of the Shafarevich-Tate group and the group of rational points for some modular abelian varieties, Algebra i Analiz 1 (1989), no. 5, 171-196. MR 91c:11032

[KL92] V.A. Kolyvagin and D. Y. Logachev, Finiteness of IU over totally real fields, Math. USSR Izvestiya 39 (1992), no. 1, 829-853. MR93d:11063

[KS00] D. R. Kohel and W. A. Stein, Component Groups of Quotients of $J_{0}(N)$, Proceedings of the 4th International Symposium (ANTS-IV), Leiden, Netherlands, July 2-7, 2000 (Berlin), Springer, 2000.

[Lan91] S. Lang, Number theory. III, Springer-Verlag, Berlin, 1991, Diophantine geometry. MR 93a:11048

[LO85] H. W. Lenstra, Jr. and F. Oort, Abelian varieties having purely additive reduction, J. Pure Appl. Algebra 36 (1985), no. 3, 281-298. MR86e:14020

[LS02] Joan-C. Lario and René Schoof, Some computations with Hecke rings and deformation rings, Experiment. Math. 11 (2002), no. 2, 303-311, with an appendix by Amod Agashe and William Stein. MR2004b:11072

[Maz72] B. Mazur, Rational points of abelian varieties with values in towers of number fields, Invent. Math. 18 (1972), 183-266. MR56:3020

[Maz77] B. Mazur, Modular curves and the Eisenstein ideal, Inst. Hautes Études Sci. Publ. Math. (1977), no. 47, 33-186 (1978). MR.80c:14015

[Maz78] B. Mazur, Rational isogenies of prime degree (with an appendix by D. Goldfeld), Invent. Math. 44 (1978), no. 2, 129-162. MR80h:14022

[MT74] B. Mazur and J. Tate, Points of order 13 on elliptic curves, Invent. Math. 22 (1973/74), 41-49. MR:50:327

[Mil86] J. S. Milne, Abelian varieties, Arithmetic geometry (Storrs, Conn., 1984), Springer, New York, 1986, pp. 103-150.

[Ogg73] A. P. Ogg, Rational points on certain elliptic modular curves, Analytic number theory (Proc. Sympos. Pure Math., Vol XXIV, St. Louis Univ., St. Louis, Mo., 1972), Amer. Math. Soc., Providence, R.I., 1973, pp. 221-231. MR 49:2743 
[PS99] B. Poonen and M. Stoll, The Cassels-Tate pairing on polarized abelian varieties, Ann. of Math. (2) 150 (1999), no. 3, 1109-1149. MF2000m:11048

[Shi73] G. Shimura, On the factors of the jacobian variety of a modular function field, J. Math. Soc. Japan 25 (1973), no. 3, 523-544. MR47:6709

[Shi94] G. Shimura, Introduction to the arithmetic theory of automorphic functions, Princeton University Press, Princeton, NJ, 1994, Reprint of the 1971 original, Kan Memorial Lectures, 1. MR95e:11048

[Ste82] G. Stevens, Arithmetic on modular curves, Birkhäuser Boston Inc., Boston, Mass., 1982. MR:87b:11050

[Ste00] W. A. Stein, Explicit approaches to modular abelian varieties, Ph.D. thesis, University of California, Berkeley (2000).

[Ste02a] W. A. Stein, An introduction to computing modular forms using modular symbols, to appear in an MSRI Proceedings (2002).

[Ste02b] W. A. Stein, Shafarevich-Tate groups of nonsquare order, Proceedings of MCAV 2002, Progress of Mathematics (to appear).

[Stu87] J. Sturm, On the congruence of modular forms, Number theory (New York, 1984-1985), Springer, Berlin, 1987, pp. 275-280. MR 88h:11031

[Tat63] J. Tate, Duality theorems in Galois cohomology over number fields, Proc. Internat. Congr. Mathematicians (Stockholm, 1962), Inst. Mittag-Leffler, Djursholm, 1963, pp. 288-295. MR 31:168

[Tat66] J. Tate, On the conjectures of Birch and Swinnerton-Dyer and a geometric analog, Séminaire Bourbaki, Vol. 9, Soc. Math. France, Paris, 1966 (reprinted in 1995), Exp. No. 306, 415-440.

Department of Mathematics, University of Texas, Austin, Texas 78712

E-mail address: agashe@math.utexas.edu

Department of Mathematics, Harvard University, Cambridge, Massachusetts 02138

E-mail address: was@math.harvard.edu

Division of Pure Mathematics, School of Mathematical Sciences, University of NotTINGHAM, ENGLAND

E-mail address: john.cremona@nottingham.ac.uk

Department of Mathematics, Harvard University, Cambridge, Massachusetts

E-mail address: mazur@math.harvard.edu 